archives-ouvertes

\title{
Physics of trap assisted photomultiplication in vertical organic photoresistors
}

\author{
Mehdi Daanoune, Raphael Clerc, Bruno Flament, Lionel Hirsch
}

\section{To cite this version:}

Mehdi Daanoune, Raphael Clerc, Bruno Flament, Lionel Hirsch. Physics of trap assisted photomultiplication in vertical organic photoresistors. Journal of Applied Physics, American Institute of Physics, 2020, 127 (5), pp.055502. 10.1063/1.5126338 . hal-02469265

\section{HAL Id: hal-02469265 \\ https://hal.archives-ouvertes.fr/hal-02469265}

Submitted on 6 Feb 2020

HAL is a multi-disciplinary open access archive for the deposit and dissemination of scientific research documents, whether they are published or not. The documents may come from teaching and research institutions in France or abroad, or from public or private research centers.
L'archive ouverte pluridisciplinaire HAL, est destinée au dépôt et à la diffusion de documents scientifiques de niveau recherche, publiés ou non, émanant des établissements d'enseignement et de recherche français ou étrangers, des laboratoires publics ou privés. 


\title{
Physics of Trap Assisted Photo-Multiplication in Vertical Organic Photoresistors
}

\author{
Mehdi Daanoune ${ }^{\mathrm{a}}$, Raphaël Clerc*a, Bruno Flament ${ }^{\mathrm{b}}$, and Lionel Hirsch ${ }^{\mathrm{b}}$. \\ a) Institut d'Optique Graduate School, Université de Lyon, UJM-Saint-Etienne CNRS UMR \\ 5516, Laboratoire Hubert Curien, 42023 Saint-Etienne, France \\ b) Univ. Bordeaux, IMS, CNRS, UMR 5218, Bordeaux INP, ENSCBP, F-33405 Talence, \\ France \\ * corresponding author: raphael.clerc@institutoptique.fr.
}

\begin{abstract}
Several experimental groups have reported recently an intriguing high level of gain (PhotoMultiplication) in vertical organic photo-resistance (as well as in other technologies, such as perovskite for instance). This mechanism is sometimes named as "Trap-Assisted Photo-Multiplication". This paper investigates the origin of this mechanism by the mean of drift diffusion simulations, analytical theory and experiments, considering the particular case of $\mathrm{PCDTBT:PC}{ }_{60} \mathrm{BM}$ photoresistors, although some conclusions are likely to apply in other technologies.

It turns out that an excess of charges (induced by electron-holes carrier generation) may trigger additional carrier injection, leading to photomultiplication, under specific circumstances. We call this mechanism "gain by injection enhancement". Electron (resp. hole) trapping for P only (resp. N only) device can play this role efficiently. As these additional carriers came from contacts, significant dark current injection is thus needed to achieve large value of gain, explaining why this mechanism can occur only in $\mathrm{P}$ (or $\mathrm{N}$ ) only photoresistors (and not photodiodes or intrinsic photoresistor, i.e. with mid gap contacts). In such devices however, the detectivity remains intrinsically limited by the high level of dark injection currents required to get gain, and consequently this type of device may be interesting in particular technologies where it is not possible to achieve low dark currents using photodiodes. However, penalized by the slow traps dynamic, the cut off frequency of these devices remains extremely low $(<100 \mathrm{~Hz})$. Also, this gain takes high value only at low irradiance, making photoresistor responsivity light dependent.

All these results bring new light in analysing and optimising photoresistor, opening a large field of investigation to take advantage of gain by injection enhancement.

\section{1) Introduction}

Organic semiconductors are attractive materials for optoelectronics applications. One of the major asset of this technology is that they can be printed onto flexible, large area substrates at room temperature - a process that is both low cost and highly scalable for organic solar cells ${ }^{1,2}$ or photodiodes arrays $s^{3,4,5}$. In particular, organic photodiodes based on the bulk heterojunction concept ${ }^{6}$, thanks to the use of printing processes, allows the realization of large area imagers ${ }^{7}$, potentially flexible, at reasonable cost, featuring detectivity comparable to silicon detectors in the visible range ${ }^{8}$. In the meantime, the reliability and the cut-off frequency, the typical weak points of organic photodiodes, has also drastically been improved ${ }^{8}$. All these progresses have made possible to design and produce innovative devices for specific applications in the field of $\mathrm{X}$ ray imaging ${ }^{4}$, human-machine interaction, and fingerprint recognition for instance.
\end{abstract}


To date, most of organic photodetectors are essentially vertical photodiodes architecture where the active layer is sandwiched between asymmetric electrodes with different work functions ${ }^{9}$. I-V characteristics are then obtained without intentional doping like in classical PN or PIN silicon photodiodes. One can also use two electrodes with similar work functions to perform organic photoconductors ${ }^{3}$ (also called photoresistors or photo conductive cells). These devices have the combined advantages to potentially feature gain (also called photo-multiplication), i.e. to collect more than one electron for one photon absorbed. Nevertheless, this architecture give rise to larger dark current and lower cut-off frequency ${ }^{3}$. Cadmium-Sulfide photoconductors are for instance currently extensively used for low cost IR detection ${ }^{10}$.

Recently, a significant number of experimental papers ${ }^{11,12}$ have reported photoconductors featuring a huge value of the gain (record value $\approx 1000^{11}$ ) in organic based devices as well in as other materials, such as perovskite ${ }^{13,14,15}$ for instance. These results are intriguing for several reasons. Firstly, in most papers, a large gain is obtained typically when defects, potentially operating like traps, are intentionally introduced. This mechanism is referred sometimes as "TrapAssisted Photo-multiplication"16 ${ }^{17}{ }^{18}$. These defects can be either $\mathrm{C}_{60}{ }^{19}$, nano-particles such as $\mathrm{ZnO}^{20}$, $\mathrm{PbSe}^{19}, \mathrm{PbS}^{21,22}{ }_{23}, \mathrm{CdSe}^{24}, \mathrm{CdTe}^{25}$, variation of the polymer/PCBM blend ratio ${ }^{18,26,27}$, or interface charge trapping layer $^{12}$. This observation may seem counterintuitive, as trapping or recombination, penalizing the collection of photo-generated carriers, are usually avoided in photo-detection. Secondly, as simulations have not been used so far to confirm the value of the measured gain, its physical origin remains unclear and subject to speculation. For instance, some authors attribute the gain to the presence of volume traps ${ }^{19}$, other to interface quality ${ }^{28}$, combining either interface traps or injection controlled by tunneling ${ }^{12}$. Moreover, the gain has been observed essentially in vertical structures (as illustrated in Figure 1), while conventional crystalline photoconductors are planar, and collect the charges by lateral contacts ${ }^{29}$. In consequence, the classical theory of photo-conductance ${ }^{29,30}$ (which treats separately the transport of photo-generated carriers in the horizontal direction and the carrier generation by light in the vertical direction) is not supposed to apply in vertical device. In fact, even if this theory is sometimes claimed to explain experiments ${ }^{19-26}$, there are evidence of incoherency between the classical theory of photoconductance and experiments, such as the unexpected decrease of gain with light intensity ${ }^{17,21,24}$ or its dependency with temperature ${ }^{11}$.

This paper proposes, based on Drift Diffusion theory, an original explanation of the origin of gain in vertical photoconductor. In particular, it demonstrates that light absorption can trigger charges injection by the contacts (a mechanism that is named in this paper "gain by injection enhancement") and discuss the conditions required to obtain this effect. It also explains why this phenomenon is much more pronounced at low light intensity, making the responsivity dependent of the light flux. In addition, the role of trapping as a booster of gain is analyzed. This theory is in very good agreement with new experiments performed on organic photoconductors. Moreover, the impact of gain on overall device performances (detectivity and cut-off frequency) is also discussed. Finally, several original solutions to enhance further the detectivity of vertical photoconductor are investigated. This work considers exclusively the case of organic photodetector with metallic contacts, even if we believe that it can be easily extended to other kind of materials or contacts, provided that specific effects, such as ionic transport in the case of perovskite material, or tunneling in the case of insulating contacts, are included. 


\section{2) Investigation of the main features of gain in organic photodetectors by numerical simulations}

In this first section, the main features of gain in both organic photoconductors and photodiodes are investigated by Drift-Diffusion simulations. Details about devices, materials parameters and models used in the simulation are presented first, before discussion.

a) Description of the template devices and model
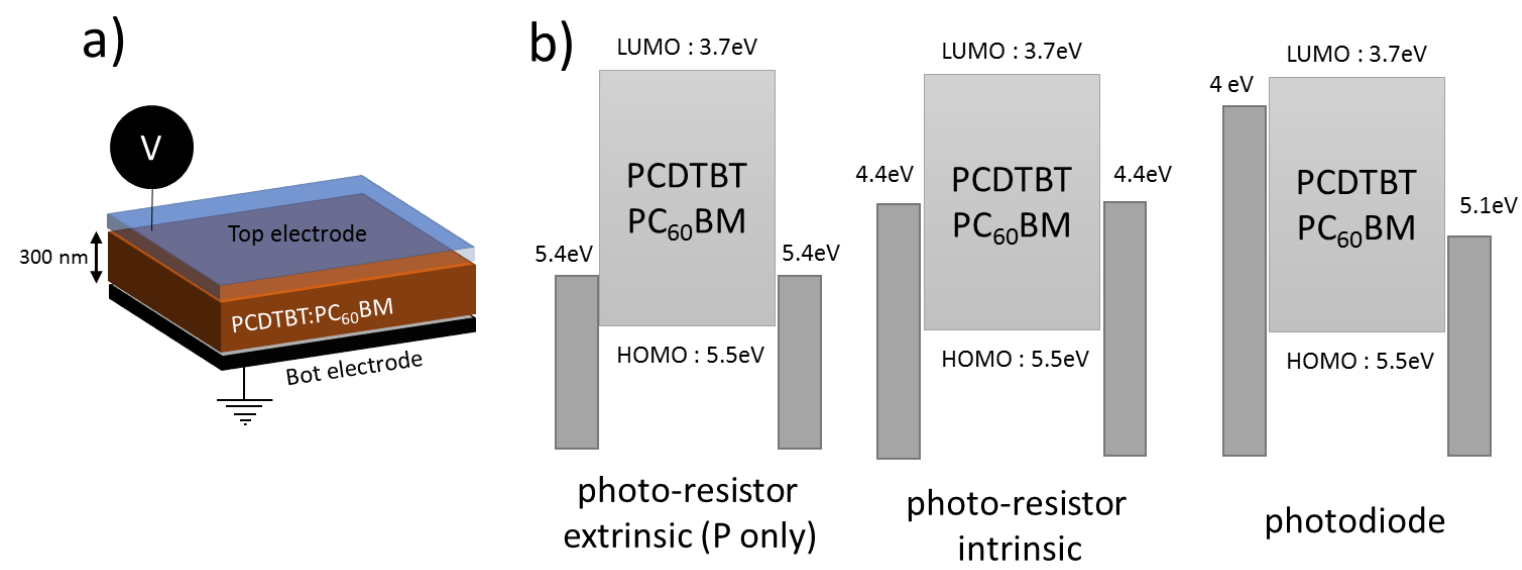

Figure 1: a) Schematic view of the organic photo-resistance considered in this section. b) Energy diagram of the PCDTBT:PC ${ }_{60} B M$ photo-resistance at equilibrium and zero bias

The device structures of the organic photodetectors considered in this work are depicted in Figure 1. In all devices, the photoactive layer is a poly(2,7-carbazole-alt-4,7-dithienyl-2,1,3-benzothiadiazole) (PCDTBT) and phenyl-C61-butyric acid methyl ester (PC60BM) PCDTBT:PC 60 BM bulk heterojunction, materials known to lead to overall good device performances and well documented in the literature ${ }^{8}$. The blend was treated as a homogeneous semiconductor, following the approach proposed by Koster et al. ${ }^{31}$. Three typical template device architectures were considered. The first one is a photoconductor with symmetrical hole injecting contacts with electrode work function at c.a. -5.4 eV, typically the poly(3,4-ethylenedioxythiophene) polystyrene sulfonate (PEDOT:PSS). This architecture leads to a "hole-only" or "p-only" device. For the second architecture, mid gap symmetrical contacts with electrode work function at c.a. $-4.4 \mathrm{eV}$ (typically Aluminum or ITO with PEIE (Polyethylenimine ethoxylated ${ }^{32}$ )) have been used. The third architecture is a photodiode with asymmetric contacts. It combines each of the previously mentioned contacts for comparison, i.e. one hole and one electron injecting contact (even though a better electron injecting contact would have been used in typical photodiode, however without affecting the generality of the results). In analogy with doped crystalline photoconductors, the "p-only" device is called extrinsic photoconductor, while the device with mid gap contact is called intrinsic photoconductor (even if in our case, no doping was used).

The transport of injected and photo-generated carriers within the device was modelled through the Drift-Diffusion approximation, which has been shown by several authors ${ }^{9,31,33-35}$ to model efficiently either injection, transport, generation or trapping of carriers both in the dark and illuminated regimes. The numerical simulations were performed using the Fluxim software. ${ }^{36}$ It allows the introduction of experimentally determined parameters e.g. electrode work function, charge carrier mobility, etc. 
Importantly, the classical boundary conditions introduced by Schottky ${ }^{29}$ were used, which consists in keeping the electron and hole concentrations at electrode contacts constant under field and lighting and equal to their equilibrium values. Previous results have shown that these boundary conditions were in good agreement with experiments, accurately predicting the impact of contact work function modification and temperature ${ }^{37}$. Moreover, the HOMO and LUMO effective density of states $\mathrm{N}_{0}$ was selected according to a common value found in literature ${ }^{38}$ to $10^{26} \mathrm{~m}^{-3}$. The blend material parameters and thickness $(300 \mathrm{~nm})$ are kept equal in the three devices and are reported in Table 1.

As in PCDTBT:PC 60 BM bulk heterojunction, the mobility of electrons and holes have been found of the same order of magnitude ${ }^{39}$ and in order to simplify the discussion on the origin of gain, both electron and hole mobilities were also taken constant and equal to $5 \times 10^{-3} \mathrm{~cm}^{2} / \mathrm{V}$.s. Of course, it is also possible to consider different mobility models for electrons and for holes and their temperature and electric field dependency. It will be done later on in this paper.

As it has been suggested in the literature that the gain was impacted by electron trap levels lower than the LUMO level (shallow trap), simulations were performed with and without shallow traps. In many organic photodiodes (including PCDTBT:PC ${ }_{60} \mathrm{BM}$ blends), these traps are typically observed in oxygen contaminated bulk heterojunction ${ }^{37}{ }^{40,41}$. In absence of light, these traps are empty. In presence of light, photo generated electron may remain trapped within the traps, creating negative charges within the active layer of the device. The $C_{n}, C_{p}$ trap parameters are not accurately known, and a large set of value can be found in the literature ${ }^{37,42}$. We decided arbitrary to use the value of $10^{-13} \mathrm{~cm}^{3} / \mathrm{s}$. However, note that the recombination lifetime $\left(\left(C_{n}+C_{p}\right) N_{T}\right)^{-1}$ considered in this work for trap concentration in the range of $10^{17}-10^{19} \mathrm{~cm}^{-3}$ are in good agreement with experimental measurements $(50 \mu \mathrm{s}-0.5 \mu \mathrm{s})^{42,43}$.

All device considered are vertical devices, where the light is entering through one semitransparent contact (and not laterally in conventional crystalline photoconductors). To model the light propagation in the stack, the optical indexes of the PCDTBT:PC ${ }_{60} \mathrm{BM}$ were used. These values were extracted from previous reflectance and transmittance experiments, following the approach proposed $\mathrm{in}^{44}$. The monochromatic light source was set $(430 \mathrm{~nm})$ with a constant power of $0.1 \mathrm{~mW} /$ $\mathrm{cm}^{2}$. This wavelength was chosen to lead to a globally uniform generation in the organic blend of $300 \mathrm{~nm}$ thickness in order to simplify the first step of the discussion. When comparing with experiments (section 4), a green LED was used.

Finally yet importantly, each photon absorbed in the blend is supposed to lead to the creation of a dissociated electron-hole pair, which, of course, is a very optimistic assumption, because of the difficulty of dissociating excitons in the real blend.

\begin{tabular}{|l|l|}
\hline \multicolumn{2}{|c|}{ Global parameters } \\
\hline HOMO & $3.7 \mathrm{eV}$ \\
LUMO & $5.5 \mathrm{eV}$ \\
$N_{0}$ & $10^{26} \mathrm{~cm}^{-3}$ \\
Mobility $\left(\mu_{n}=\mu_{p}\right)$ & $5 \times 10^{-3} \mathrm{~cm}^{2} / \mathrm{V} . \mathrm{s}$ \\
Work function & Between $4.4 \mathrm{eV}$ and $5.36 \mathrm{eV}$ \\
Wavelength & $430 \mathrm{~nm}($ corresponding to an absorbance of 0.842$)$ \\
Intensity power & $0.1 \mathrm{~mW} / \mathrm{cm}^{2}$ \\
Length/thickness & $300 \mathrm{~nm}$ \\
\hline \multicolumn{2}{|c|}{ Trap parameters (electron acceptor) } \\
\hline$E_{T}$ & $0.7 \mathrm{below}$ the LUMO level \\
$N_{T}$ (N type) & $1 \times 10^{19} \mathrm{~cm}^{-3}$ \\
Capture rate: $\mathrm{Cp}, \mathrm{Cn}$ & $1 \times 10^{-13} \mathrm{~cm}^{3} / \mathrm{s}$ \\
\hline
\end{tabular}


Table 1 : Summary of the simulation parameters used in this section.

b) Results: Comparison between photodiodes and photoresistor

Main results are summarized in Table 2. As expected, the defect-free photodiode has the lower level of dark current, followed by the defect-free intrinsic photo-resistance and the photodiode with traps. Extrinsic photoresistor, with or without traps, have a larger dark current. Note that the value of the dark current are (as expected) largely dependent of the contact nature.

More surprising, only the extrinsic photo-resistance shows significant photo-multiplication (gain larger than unity). This gain is very important in presence of traps ( 1150), in agreement with previous experimental observation in the literature (see for instance the review ${ }^{16}$ ). However, a gain is also present in absence of trap, even though at very modest level ( 2). Clearly, this low value of gain has certainly low interest for application.

However, the observation of gain in absence of traps is interesting from a fundamental point of view, as it proves that photomultiplication is not the direct consequence of trapping. In addition, it cannot be explained with the classical theory of gain ${ }^{29}$ in conventional planar photo-resistance. Indeed, according to this model, the gain is equal to:

$$
\text { Gain }_{\text {class }}=\frac{\tau}{\tau_{\text {coll }}}
$$

Where $\tau$ is the charge carrier lifetime due to recombination, and $\tau_{\text {coll }}$ the collection or transit time, given by:

$$
\tau_{\text {coll }}=\left(\frac{\mu_{n} V_{a}}{L^{2}}+\frac{\mu_{p} V_{a}}{L^{2}}\right)^{-1}
$$

Where $\mu_{n}$ (resp. $\mu_{p}$ ) is the electron (resp. hole) mobility, $L$ is the active layer thickness, $V_{a}$ the applied voltage. In absence of recombination ( $\tau$ tends to the infinite), the gain Gain class $_{\text {should diverge, }}$ suggesting that this theory does not apply in absence of recombination.

In conclusion, simulations confirm that photo-multiplication may occur extrinsic photo-resistance. This mechanism is particularly strong in presence of electrons traps (for hole only photo-resistance), but may exist also in principle in absence of any traps. This result suggests that traps only amplifies

\begin{tabular}{|c|c|c|c|c|c|c|}
\hline & Notation & $\begin{array}{c}\text { Dark } \\
\text { current } \\
\left(\mathrm{mA} / \mathrm{cm}^{2}\right)\end{array}$ & $\begin{array}{c}\text { Electron } \\
\text { current } \\
\left(\mathrm{mA} / \mathrm{cm}^{2}\right)\end{array}$ & $\begin{array}{c}\text { Hole } \\
\text { current } \\
\left(\mathrm{mA} / \mathrm{cm}^{2}\right)\end{array}$ & $S(A / W)$ & Gain \\
\hline Photodiode & $P D$ & $10^{-11}$ & 0 & $10^{-11}$ & 0.287 & 0.982 \\
\hline Photodiode with trap & $P D(T)$ & $10^{-10}$ & 0 & $10^{-10}$ & 0.117 & 0.4 \\
\hline intrinsic photo-resistance no trap & $P R$ (int) & $9.2 \times 10^{-6}$ & $9.2 \times 10^{-6}$ & $1.8 \times 10^{-12}$ & 0.282 & 0.966 \\
\hline $\begin{array}{c}\text { intrinsic photo-resistance with } \\
\text { trap }\end{array}$ & $P R$ (int)T & $10^{-10}$ & 0 & $10^{-10}$ & 0.06 & 0.2 \\
\hline extrinsic photo-resistance no trap & $P R($ ext $)$ & 318 & 0 & 214 & 0.6 & 1.93 \\
\hline $\begin{array}{c}\text { extrinsic photo-resistance with } \\
\text { trap }\end{array}$ & $P R(e x t) T$ & 318 & 0 & 318 & 336 & 1150 \\
\hline
\end{tabular}
photo-multiplications, but are not necessarily required for it to occur.

Table 2: Summary of the results of the simulations of the first section, for simplified PCDTBT:PC ${ }_{60} B M, 300 \mathrm{~nm}$ thick, photodiode, intrinsic and extrinsic photo-resistance considered at $-2 \mathrm{~V}$ with a constant illumination of $430 \mathrm{~nm}$ set at $0.1 \mathrm{~mW} / \mathrm{cm}^{2}$, traps located at $0.7 \mathrm{eV}$ below the LUMO level with a density of $10^{19} \mathrm{~cm}^{-3}$, and a constant mobility of $0.005 \mathrm{~cm}^{2} / V$.s (equal for electrons and holes). The complete simulation parameters are summarized in Table 1. 
c) Results: Impact of light irradiance on the gain of extrinsic photo-resistance.

In order to get more insight on the photo-multiplication effect, the impact of the irradiance level on the value of gain was investigated for the three device architectures mentioned above (Figure 2).

Devices with no traps and no photo-multiplication (gain closed to unity), i.e. the trap free photodiodes and intrinsic photo-resistances, does not show, as expected, any irradiance dependency. In presence of traps, the responsivity of these devices is reduced, and further degraded when irradiance increases, until reaching a constant level. This effect is expected, as it is consistent with the progressive process of trap filling until trap saturation.

Interestingly, for device featuring photo-multiplication, the gain is constant at low level of irradiance and then decreases whenever traps are present or not (even if this droop is much less pronounced for the photo-resistance without traps). This trend is intriguing, as the gain decreases despite the fact that traps are more and more filled by photogeneration. It has already been observed in several previous experimental papers ${ }^{17,21,23,24}$.

In any case, a high value of responsivity, and its decrease with light irradiance can be seen as an indication of the presence of gain, in agreement with the theory discussed in this paper, allowing to discriminate from other potential source of gain. In particular, the classical theory of gain in conventional planar photo-resistance does not predict any similar irradiance level dependency.

Even if it is also based on a drift diffusion approach, we believe that the classical theory of gain in planar photo-resistance does not apply in vertical devices. In fact, this analytical theory explicitly assumes that the conductance in presence of light remains the same for all applied voltage, which may be true when the light does not enter through the electrodes, but by a lateral side. In our case however, simulations are not based on any a-priori approximation and leads, as discussed, to different results.

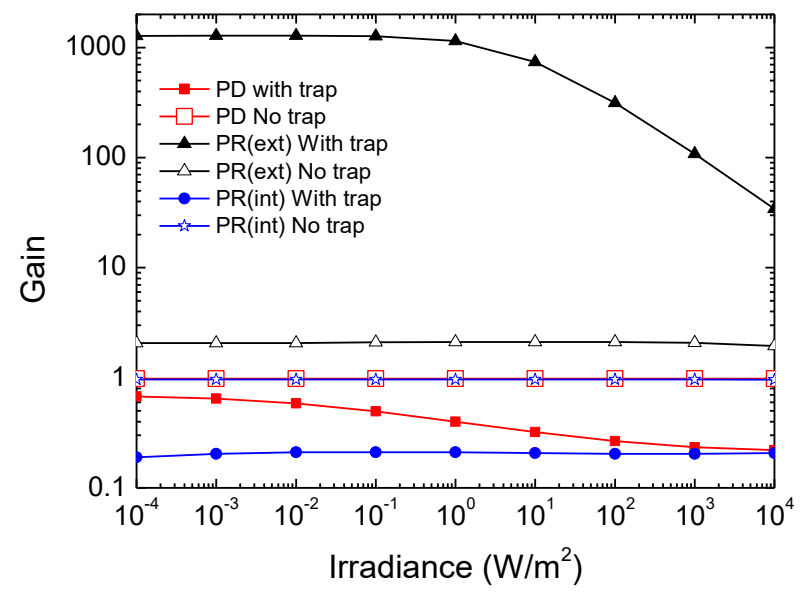

Figure 2: Gain versus the irradiance for a PCDTBT:PC ${ }_{60} B M$ different structure at $-2 \mathrm{~V}$. The simulation was performed for a monochromatic illumination set at $430 \mathrm{~nm}$. The illumination intensity power varies from $10^{-4} \mathrm{~W} / \mathrm{m}^{2}$ to $10^{4} \mathrm{~W} / \mathrm{m}^{2}$. The other simulation parameters are kept identical to those used in the previous section and are reported in Table 1.

The gain is also voltage and wavelength dependent, as discussed in the supplementary materials. 


\section{3) Theory of gain by injection enhancement: model and discussion}

As seen previously, steady state drift diffusion simulations confirm the existence of photomultiplication, however it is not obvious just from simulation to get detailed insight on the origin of the phenomenon. Indeed, as drift and diffusion equations compute the total electron and hole concentration at each point, it is not possible to discriminate from the direct result of calculations carriers that have been directly photo-generated by photon absorption from other carriers induced by photo-multiplication.

For this reason, a model is proposed in this section, allowing to investigate the origin of photomultiplication. Precious indications can be obtained from this model, as seen later. According to the calculations detailed in appendix A, it is possible to get an expression of the gain (noted "Gain" to avoid confusion with the generation rate $G$ ) in the framework of the drift diffusion model, without any approximations. This expression is:

$$
\text { Gain }=\left|\frac{<\phi_{G}(x)-\phi_{R}(x)>_{i}-<\phi_{R d}(x)>_{d}}{\phi_{G t}}+\frac{-\beta_{n} \tilde{\phi}_{n d}+\beta_{p} \tilde{\phi}_{p d}}{\phi_{G t}}\right|
$$

where $\tilde{\phi}_{n d}$ (resp. $\tilde{\phi}_{p d}$ ) is the electron (resp. hole) carrier flux in dark condition, $\phi_{G t}$ is the photon flux $\left(\mathrm{m}^{-2} \mathrm{~s}^{-1}\right)$ absorbed in the active layer (thickness $\left.\mathrm{L}\right)$, and

$$
\begin{gathered}
\phi_{G}(x)=\int_{0}^{x} G\left(x^{\prime}\right) d x^{\prime} \\
\phi_{R}(x)=\int_{0}^{x} R\left(x^{\prime}\right) d x^{\prime} \\
\phi_{G t}=\phi_{G}(L)=\int_{0}^{L} G\left(x^{\prime}\right) d x^{\prime} \\
<\phi_{G}(x)-\phi_{R}(x)>_{i} \\
=-\frac{\int_{0}^{L}\left[\phi_{G}(x)-\phi_{R}(x)\right] \exp \left(\frac{e V(x)}{k T}\right) d x}{\int_{0}^{L} \exp \left(\frac{e V(x)}{k T}\right) d x} \\
+\frac{\int_{0}^{L}\left[\phi_{G}(x)-\phi_{R}(x)\right] \exp \left(-\frac{e V(x)}{k T}\right) d x}{\int_{0}^{L} \exp \left(-\frac{e V(x)}{k T}\right) d x} \\
\left.<\phi_{R d}(x)>_{d}=\frac{\int_{0}^{L} \phi_{R d}(x) \exp \left(\frac{e V_{d}(x)}{k T}\right) d x}{\int_{0}^{L} \exp \left(\frac{e V(x)}{k T}\right) d x}-\frac{e V_{d}(x)}{k T}\right) d x
\end{gathered}
$$

$\mathrm{G}$ (resp. $\mathrm{R}$ ) is the generation (resp. recombination) rate under illumination. See the appendix for the definition of other parameters. This formula allows to recalculate the gain using the electrostatical potential $V$ and $V_{d}$ extracted from simulation in very good agreement with direct simulation results (see Table 3), and more importantly to identify its different contributions, as discussed in the following. However, let us stress that equation (3) cannot allow to calculate the gain alone, as the potential profile $V$ under illumination and $V_{d}$ under dark are not known, and have to be extracted from simulations. The aim of this model is not to calculate the gain, but rather to help understanding simulation results, as detailed in the following. A complete analytical model of gain would require formula for $V(x)$ and $V_{d}(x)$, but this is beyond the scope of this paper.

\begin{tabular}{|l|l|l|c|c|c|c|c|c|}
\hline & $\alpha$ & $\beta_{\mathrm{e}}$ & $\begin{array}{c}\text { Electron } \\
\text { dark } \\
\text { current }\end{array}$ & $\beta_{\mathrm{h}}$ & $\begin{array}{c}\text { Hole dark } \\
\text { current } \\
\left(\mathrm{mA} / \mathrm{cm}^{2}\right)\end{array}$ & $\begin{array}{c}\widetilde{\phi}_{n d} / \phi_{G t} / \phi_{G t} \\
\widetilde{\phi}_{\boldsymbol{p}}\end{array}$ & $\begin{array}{c}\text { Gain } \\
\text { (Simu.) }\end{array}$ & $\begin{array}{c}\text { Gain } \\
\text { Eq. (3) }\end{array}$ \\
\hline
\end{tabular}




\begin{tabular}{|c|c|c|c|c|c|c|c|c|}
\hline & & (mA/cm $)$ & & & & & \\
\hline Photodiode & 0.98 & $-4 \times 10^{-6}$ & 0 & $3 \times 10^{-5}$ & $-10^{-11}$ & $\begin{array}{c}0 \\
10^{-11}\end{array}$ & 0.98 & 0.98 \\
\hline $\begin{array}{c}\text { Photodiode with } \\
\text { trap }\end{array}$ & 0.41 & -0.451 & 0 & 0.8 & $-10^{10}$ & $\begin{array}{c}0 \\
5 \times 10^{-9}\end{array}$ & 0.4 & 0.41 \\
\hline $\begin{array}{c}\text { photo-resistance } \\
\text { intrinsic (No trap) }\end{array}$ & 0.97 & $-10^{-5}$ & $9 \times 10^{-6}$ & $6 \times 10^{-5}$ & $10^{-12}$ & $\begin{array}{c}3 \times 10^{-4} \\
6 \times 10^{-11}\end{array}$ & 0.966 & 0.97 \\
\hline $\begin{array}{c}\text { photo-resistance } \\
\text { extrinsic (No trap) }\end{array}$ & 0.93 & $4 \times 10^{-7}$ & 0 & $2 \times 10^{-5}$ & 214 & $\begin{array}{c}0 \\
5 \times 10^{4}\end{array}$ & 1.93 & 1.93 \\
\hline $\begin{array}{c}\text { photo-resistance } \\
\text { intrinsic (Trap) }\end{array}$ & 0.20 & $5 \times 10^{-5}$ & 0 & -1 & $5 \times 10^{-9}$ & $\begin{array}{c}0 \\
5 \times 10^{-9}\end{array}$ & 0.2 & 0.20 \\
\hline $\begin{array}{c}\text { photo-resistance } \\
\text { extrinsic (Trap) }\end{array}$ & 0.92 & $10^{-3}$ & 0 & 0.105 & 318 & $\begin{array}{c}0 \\
10^{4}\end{array}$ & 1150 & 1156 \\
\hline
\end{tabular}

Table 3: Summary of the result of the simulation and coefficient $\beta_{\mathrm{e}}, \beta_{\mathrm{h}}$ and $\alpha$ (The value and gain was recalculated with formula (3) and compared with data directly extracted from simulation) for a PCDTBT:PC 60 BM photodiode, photo-resistance intrinsic and photo-resistance extrinsic at $-2 \mathrm{~V}$. The other simulation parameters are kept identical to those used in the previous section reported in Table 2.

Let us consider the first term, $\alpha$, defined as:

$$
\alpha=\frac{\left|<\phi_{G}(x)-\phi_{R}(x)>_{i}-<\phi_{R d}(x)>_{d}\right|}{\phi_{G}(L)}
$$

This $\alpha$ term would correspond to the internal quantum efficiency in absence of photo-multiplication. Indeed, let us prove that $\alpha$ cannot be higher than 1 . As recombination tends to decrease $\alpha$, we have:

Where

$$
\alpha<\frac{\left|<\phi_{G}(x)>_{i}\right|}{\phi_{G}(L)}<\max (A, B)
$$

$$
\begin{array}{r}
A=\frac{\int_{0}^{L}\left[\phi_{G}(x) / \phi_{G}(L)\right] \exp \left(\frac{e V(x)}{k T}\right) d x}{\int_{0}^{L} \exp \left(\frac{e V(x)}{k T}\right) d x} \\
B=\frac{\int_{0}^{L}\left[\phi_{G}(x) / \phi_{G}(L)\right] \exp \left(-\frac{e V(x)}{k T}\right) d x}{\int_{0}^{L} \exp \left(-\frac{e V(x)}{k T}\right) d x}
\end{array}
$$

Moreover, both A and B are lower than 1, as $\phi_{G}(x)<\phi_{G}(L)$. Being lower than unity, this term cannot induce gain.

The second term, under some circumstances, may be responsible for photo-multiplication, as explained in the following. First of all, let us stress that this term is proportional to the dark current, as $\tilde{\phi}_{n d}$ (resp. $\tilde{\phi}_{p d}$ ) is the flux of electrons under dark condition (resp. the flux of holes). First interesting conclusion, the photo-multiplication process only occurs in device featuring significant dark current, which explains why photodiodes or intrinsic photo-resistance have not shown any gain in previous simulations (see Table 3). Moreover, being proportional to the dark electron or holes flux, this term represents clearly an additional source of current provided by contact injection. As discussed in the following, this condition is necessary, but not sufficient. 
As extrinsic photo-resistances are either $\mathrm{P}$ or $\mathrm{N}$ only devices (hole or electron are majority carrier), there is no need to consider both terms simultaneously. Let us consider the case of $\mathrm{P}$ only device. In this case, the gain equation simplifies:

$$
\text { Gain }=\left|\alpha+\beta_{p} \frac{\tilde{\phi}_{p d}}{\phi_{G t}}\right|
$$

The $\beta_{p}$ coefficient is given by:

$$
\beta_{p}=\frac{\int_{0}^{L} \exp \left(\frac{e V_{d}(x)}{k T}\right) d x}{\int_{0}^{L} \exp \left(\frac{e V(x)}{k T}\right) d x}-1=\frac{\int_{0}^{L} \exp \left(\frac{e V_{d}(x)}{k T}\right) d x}{\int_{0}^{L} \exp \left(\frac{e V_{d}(x)}{k T}\right) \exp \left(\frac{e \Delta V(x)}{k T}\right) d x}-1
$$

where $\Delta V(x)=V(x)-V_{d}(x)$ is the difference of electrostatic potential in presence of light $(V)$ or in absence of light $\left(V_{d}\right)$. A simple mathematical analysis of equation (14) reveals that $\beta_{p}$ is necessarily higher than -1 , and can take large positive value when $\Delta V \ll-k T$. This later case corresponds to device with large gain. However, this condition is not easy to achieve, as discussed in the following. $\Delta$ $V \neq 0$ means that light carrier generation should induce a significant modification of the charge within the device. This condition requires particular conditions to be satisfied. Indeed, it is generally assume on the contrary that there is no significant change in the total charge ${ }^{30}$ after illumination ("quasi neutrality" approximation), as both negative charges (electrons) and positive charges (holes) are generated simultaneously. The Photo-Dember effect ${ }^{29}$ is an example of known situation where $\Delta V \neq$ 0 on the contrary, due to the difference of mobility of photogenerated electrons and holes. In our case, if the difference of mobility may also indeed induce gain, the most efficient mechanism remains trapping. Indeed, traps can capture a significant amount of one type of carrier, inducing a charge difference between dark and light conditions, explaining why traps are so efficient to create gain.

A closer analysis reveals that additional requirements are needed for the charge modification induced by photogenerated carriers to create significant gain. Indeed, first $\Delta V$ need to be negative, then $\Delta V(x)$ need to take value at a suitable position (depending on $V_{d}(x)$ ), in order to contribute significantly to the integral of Eq. (14). These unexpected requirements can be understood as follow: as the photomultiplicated carriers came from the contacts, the additional electrical field induced by light absorption need to reinforce the initial dark electrical field (and not decrease it), in order to trigger injection (at least in one region of the active layer). This situation is illustrated in Figure 3 . In this figure, the excess of dark electric field $E_{d}(x,-2 V)-E_{d}(x, 0 V)$ is plotted together with the additional field induced by light $E(x,-2 V)-E_{d}(x,-2 V)$. The excess of dark field is responsible for hole injection, causing dark current $e \phi_{\bar{d}}$. It is largely negative, meaning that holes are moving from right $(x=\mathrm{L})$ to left $(x=0)$. This device features a large level of gain because light induces (by electron trapping) a large spike of negative field close to the right electrode $(x=L)$, reinforcing injection. In consequence, the spatial repartition of the generation rate (which has been considered relatively uniform so far) is expected to have an impact on the gain. An example is shown in the supplementary material.

\begin{tabular}{c|c}
\hline \multicolumn{2}{c}{ Trap parameters used in Figure $\mathbf{3}$} \\
\hline $\mathbf{E}_{\mathrm{T}}$ & $\begin{array}{c}0.7 \text { below the LUMO level } \\
\text { (electron acceptor) }\end{array}$ \\
\hline Capture rate: $\mathbf{C p}, \mathbf{C n}$ & $1 \times 10^{-13} \mathrm{~cm}^{3} / \mathrm{s}$ \\
\hline $\mathbf{N}_{\mathrm{T}}$ & $1 \times 10^{19}\left(\mathrm{~cm}^{-3}\right)$ \\
\hline $\boldsymbol{\beta}_{\mathrm{h}}$ & 0.105 \\
\hline $\boldsymbol{\beta}_{\mathrm{e}}$ & $1.3 \times 10^{-3}$ \\
\hline Gain & 1150 \\
\hline
\end{tabular}


Table 4: Summary of the trap parameters used in Figure 3.

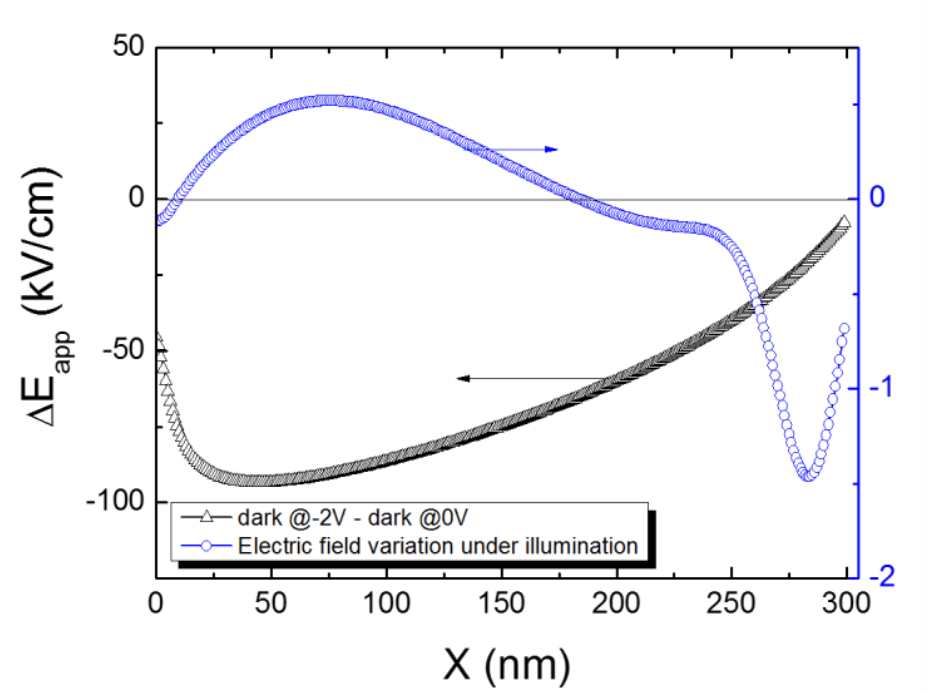

Figure 3: Simulated electric field difference between illumination and dark condition $\left(E(x)-E_{d}(x)\right)$ (blue circle). The black triangle curve corresponds to the electric field variation between $-2 \mathrm{~V}$ and $0 \mathrm{~V}$ in dark condition only. The simulation parameters are reported in Table 4.

All these explanations conclude that the photo-multiplication process is the consequence of an additional injection of majority carriers induced by electrostatic. We call it "gain by injection enhancement".

The dependency of gain with light flux (reported in Figure 2 for instance) can be understood as follow. First of all, let us note that the two terms in equation (13) depend on $\phi_{G t}$, the total number of generated electron-hole pair: the ratio $\tilde{\phi}_{p d} / \phi_{G t}$ and $\beta_{p}$. If $\beta_{p}$ linearly increase with $\phi_{G t}$, the gain is constant and if $\beta_{p}$ saturates the gain decrease as $1 / \phi_{G t}$. Keeping this in mind, the trend of gain versus irradiance can be explained as follow when trapping is involved. At low irradiance flux, traps are mostly empty and keep on charging when irradiance increases. This results on a linear increase of $\beta_{p}$ with $\phi_{G t}$, leading to a constant gain. At high irradiance however, the trap occupancy saturates, resulting in a constant $\beta_{p}$ and thus a decreasing gain.

\section{4) Experimental validation}

In order to validate our theory by comparison with experiments, $\mathrm{PCDTBT}: \mathrm{PC}_{60} \mathrm{BM}$ photoresistor have been processed, using a process very similar to the one presented in reference ${ }^{8}$. For this study, PEDOT:PSS have been used for the top $(\sim 1.5 \mu \mathrm{m})$ and bottom electrodes $(\sim 50 \mathrm{~nm})$ to have symmetric architecture. The active layer thickness was measured approximately equal to $300 \mathrm{~nm}$. For a comparison between our model and experimental data, the following field dependent mobility model has been used (assuming same mobility for electrons and holes in agreement with the literature ${ }^{39}$ ).

$$
\mu(E)=\mu_{p} \exp (B \sqrt{E})
$$

Interestingly, all parameters needed for reproducing experiments were already determined in a previous work ${ }^{37}$, by comparison between simulations and experiments on photodiodes (not photoresistor). No further adjustment was needed to reproduce photoresistor results, except a minor adjustment of the value of the work functions of PEDOT electrodes (around $5.36 \mathrm{eV}$ ). The bottom PEDOT:PSS electrode is spin-coated whereas the top PEDOT:PSS electrode is screen-printed. Moreover, the deep traps present in the simulation, and responsible for gain in this case, were natively 
present in the active layer, and already identified in reference ${ }^{37}$, in order to reproduce correctly the level on illumination current on virgin and aged devices. The same trap parameters were used for both photodiode and photoresistor simulations. In experiments, the light source was a green LED ( $536 \mathrm{~nm}$, close to the maximum sensitivity peak of PCDTBT: $\mathrm{PC}_{60} \mathrm{BM}$ photodiode ${ }^{8}$ ). The gain in these devices was found equal to $\sim 5$ at $-2 \mathrm{~V} @ 24.671 \mathrm{~W} / \mathrm{m}^{2}$ (by simulations, see Figure 4 (b)).

\begin{tabular}{|c|c|c|}
\hline \multicolumn{3}{|c|}{ Simulations parameters } \\
\hline Work function & $5.36 \mathrm{eV}$ & $5.36 \mathrm{eV}$ \\
\hline$\mu_{0}$ & \multicolumn{2}{|c|}{$\mu_{n}=\mu_{p}=8 \times 10^{-3} \mathrm{~cm}^{2} / \mathrm{V} . \mathrm{s}$} \\
\hline B & \multicolumn{2}{|c|}{$0.65 \times 10^{-2}(\mathrm{~cm} / \mathrm{V})^{0.5}$} \\
\hline$\lambda$ & \multicolumn{2}{|c|}{$536 \mathrm{~nm}$} \\
\hline Irradiance & \multicolumn{2}{|c|}{$24.671\left(\mathrm{~W} / \mathrm{m}^{2}\right)$} \\
\hline Active layer thickness & \multicolumn{2}{|c|}{$300 \mathrm{~nm}$} \\
\hline Active area & \multicolumn{2}{|c|}{$2.5 \times 10^{-6}\left(\mathrm{~m}^{2}\right)$} \\
\hline \multicolumn{3}{|c|}{ Trap parameters } \\
\hline$E_{T}(e V)$ & \multicolumn{2}{|c|}{$0.7 \mathrm{eV}$ below the LUMO level } \\
\hline $\mathbf{N}_{\mathrm{T}}$ & \multicolumn{2}{|c|}{$3 \times 10^{17}\left(\mathrm{~cm}^{-3}\right)$} \\
\hline$C_{p} / C_{n}$ & \multicolumn{2}{|c|}{$10^{-13}\left(\mathrm{~cm}^{3} / \mathrm{s}\right)$} \\
\hline
\end{tabular}

Table 5: Parameters used in simulations to reproduce photoresistor experiments

(a)

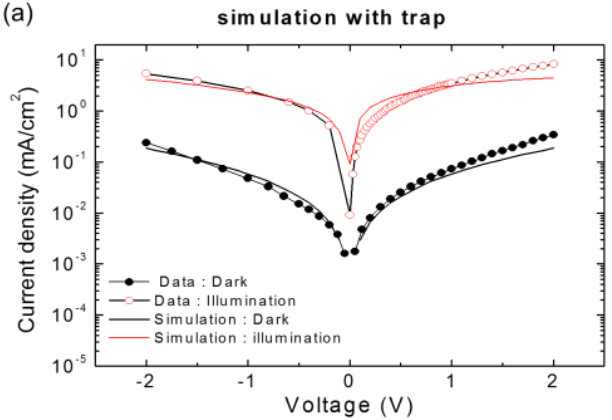

(b)

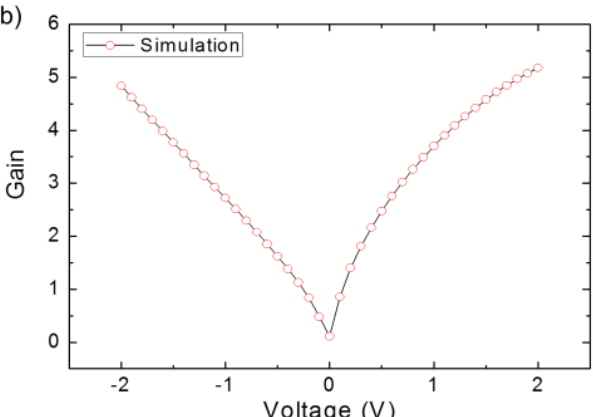

Figure 4: Illuminated and dark I-V curves, dots are experimental data, solid lines are simulations. (a) Comparison between simulation and experiments using the parameter of Table 5. (b) Corresponding calculated gain versus the voltage.

(a)

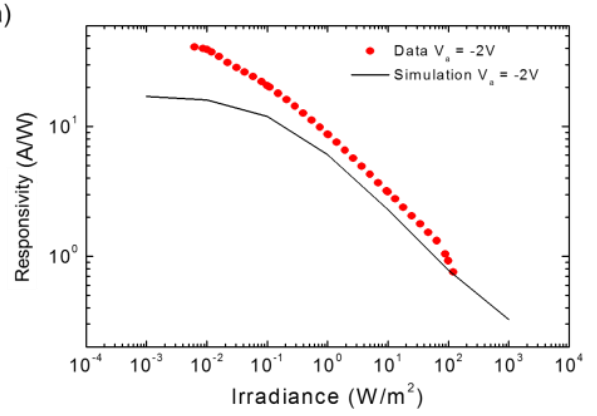

(b)

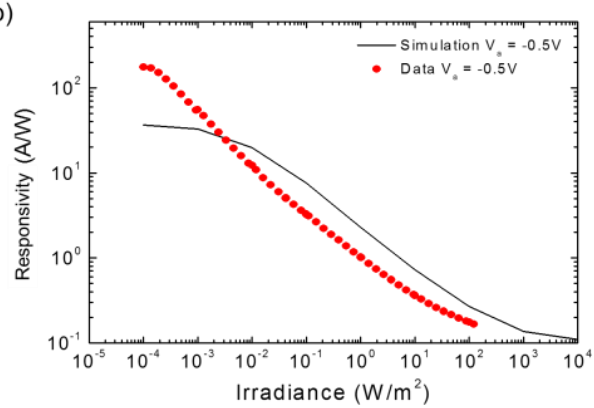

Figure 5: Responsivity versus irradiance for two different applied voltages (a) $V_{a}=-2 \mathrm{~V}$ and (b) $V_{a}$ $=-0.5 \mathrm{~V}$. Dots are experimental data, solid lines are simulations. The simulations were performed with the parameters listed in Table 5.

The agreement between experiments and simulation is satisfactory, in I-V experiments (Figure 4) as well as in responsivity versus irradiance (Figure 5), which confirms the validity on the simulation and theory discussed in the paper. In particular, the decrease of responsivity with irradiance agrees with the theoretical results presented in Figure 2. 


\section{5) Consequences on device performances}

In the previous section, the physical mechanism of gain in photoresistor by injection enhancement has been investigated. In this section, the impact of this gain on device performance is discussed and compared with other devices.

\section{a) Detectivity}

One of the main figure of merit of photodetectors is detectivity ${ }^{3} D^{*}$. A device featuring a high detectivity has typically a good responsivity and a low noise level, allowing detecting low irradiance signal. The high gain at low irradiance of photoresistor enhanced by photomultiplication is clearly an advantage for the detectivity. However, it comes with a price: as previously discussed, a high gain implies a high level of dark current, leading to a high level of dark current noise. This fact rises the following question: is there a trade-off between gain and dark current noise, allowing photoresistor to be competitive with photodiodes?

To answer this question, the detectivity of several devices has been plotted versus trap concentrations on Figure 6. Three devices with no photo-multiplication (two photodiodes and an intrinsic photoresistor) were compared with two extrinsic photoresistors presenting photo-multiplication due to trapping. Devices are essentially similar to those reported in Table 1. All devices are composed of a blend of PCDTBT:PC ${ }_{60} \mathrm{BM}$, and the traps used in the simulations were the same (volume trap uniformly distributed, electron acceptor traps, mid gap, with $\left.C n=C p=10^{-13}\left(\mathrm{~cm}^{3} / \mathrm{s}\right)\right)$.

The two photodiodes considered have the same electrodes: a hole injecting contact $-5.1 \mathrm{eV}$ and an electron injecting contact $-4 \mathrm{eV}$. However, the first one (named PD) is assumed ideal. It means that the dark current due to contact injection is extremely low. Recombination does not increase it sufficiently, and for this reason, the detectivity remains photon shot noise limited, independent of the trap concentration, extremely high and unrealistic $\left(\sim 10^{15}\right.$ Jones). The best reported organic photodiodes, as in ref $^{8}$ for instance have a more reasonable detectivity in the range $3.10^{13}$ Jones, due to an enhanced dark current induced by internal shunt. For this reason, a shunt has been included in our simulation to reproduce state of the art photodiodes ( $\mathrm{PD}(\mathrm{Rp})$ symbols). In this case, the detectivity decreases with the concentration of recombination traps, essentially because the responsivity is degraded by trapping (shunt is assumed independent of trapping).

The intrinsic photoresistor has mid gap electrodes, leading to an extremely low dark current in absence of traps. In presence of traps however, the dark current increases and the responsivity decreases, leading, as expected, to a significant degradation of the detectivity.

Finally, two extrinsic P-only photoresistors were also considered (work function $-5.1 \mathrm{eV}$ and $-5.3 \mathrm{eV}$ ). Being single carrier devices, there is no significant evolution of the dark current by recombination (no electrons in dark). However, the increase of trap concentration induces a significant increase of the gain, explaining why the detectivity improves with the trap concentration in both case. The slightly better detectivity of the $5.1 \mathrm{eV}$ photoresistor is explained by its better value of $\beta_{\mathrm{h}}$. 


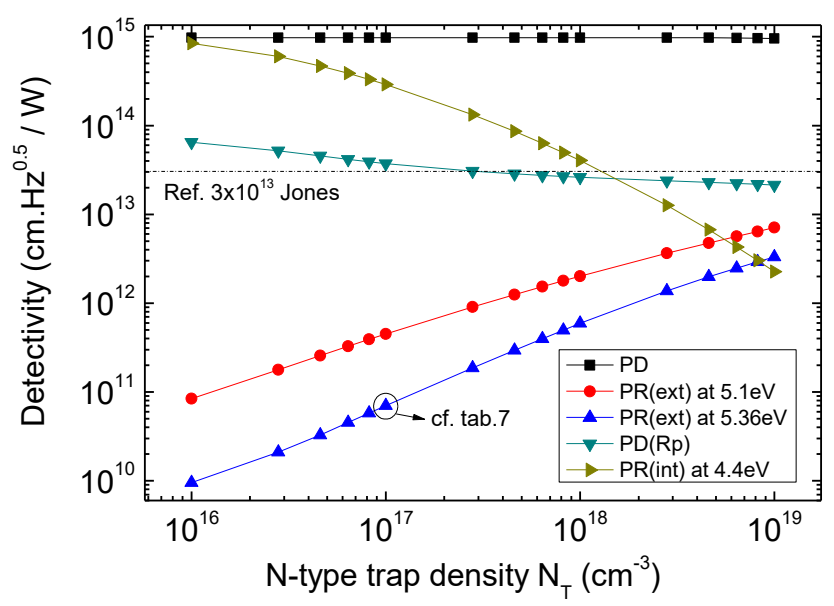

Figure 6 : Detectivity versus trap density for a PCDTBT:PC 60 BM different devices at $0.5 \mathrm{~V}$. "PD" refers to an ideal photodiode, "PD(Rp)" refers to a photodiode where the dark current is limited by a shunt resistance (see the text for details), "PR(ext)" refers to extrinsic photoresistor with gain due to traps, "PR(int)" refers to a instrinsic photoresistor (work function at $4.4 \mathrm{eV}$ ) with no gain.

The main conclusion of these simulations is that the detectivity of photoresistors can reach good value (> $10^{12}$ Jones) despite their high level of dark current. However, these theoretical performances remains lower that the best value reported in the case of state of the art visible organic photodiodes (3.10 13 Jones). In consequence, photoresistor enhanced by gain may be an interesting alternative in specific technology where it is difficult to achieve good photodiodes, either because contacts electrodes are poor, or because it is difficult to reduce traps concentration (due to impurities and disorder for instance). Also, photoresistances might be more resilient to contamination than photodiodes, as contamination might increase the traps concentration responsible for gain. However, this point has not been investigated in this work and would require further attention.

\section{b) Frequency operation}

Another figure of merit of photodetector is its bandwidth. In order to investigate the frequency operation of photo-resistance featuring photo-multiplication due to traps, simulations were performed, fixing the applied voltage, and modulating the frequency dependency of the illumination. The device considered is the same as in the experimental section (parameters listed in Table 5), including field dependent mobility.

In Figure 7, we can observe two frequency cut-offs. At about $1 \mathrm{~Hz}$, the gain rapidly drops off. It corresponds to the slow response of deep traps. Between $1 \mathrm{~Hz}$ and $1 \mathrm{MHz}$, the device has a low gain value, which approximately correspond to its gain in absence of traps. Above $1 \mathrm{MHz}$, the device has no response. It corresponds to its time of flight cut-off frequency (mobility is enhanced in this case by the large applied voltage and intrinsic RC time is quite short). As expected, the traps concentration affects the value of the steady state gain, but not its frequency operation.

Other simulations indicate that the gain frequency cut off depend on the trap level position (shallow traps are faster) but in any case, the first cut off frequency never exceed hundreds of $\mathrm{Hz}$, in agreement with experimental results reviewed in details $\mathrm{in}^{3}$.

Our simulations thus confirm that the gain by injection enhancement due to traps is a rather slow process, penalizing potential application for this type of devices. 

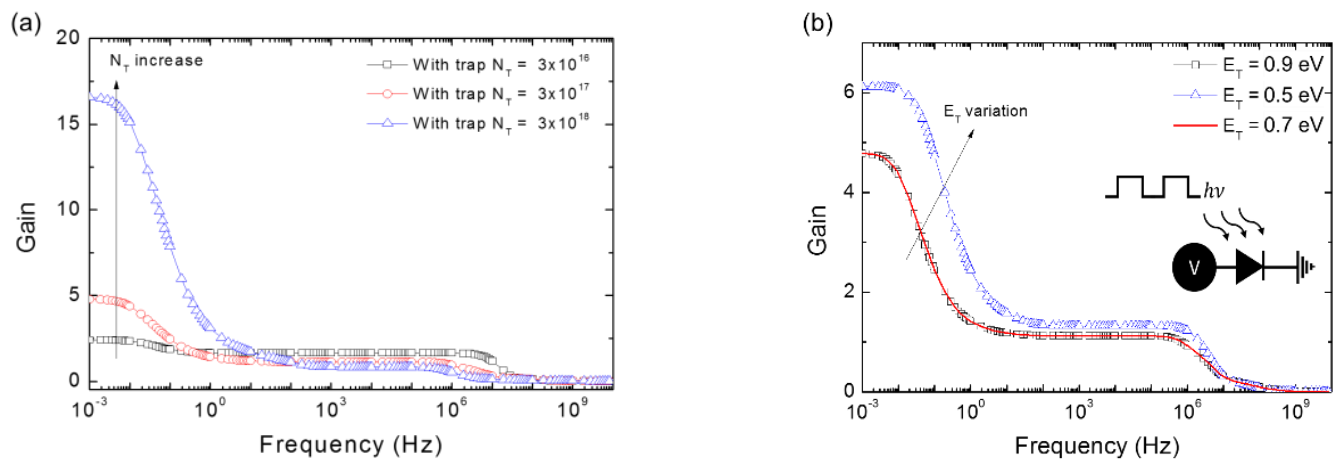

Figure 7 : Gain dependency versus frequency (applied voltage $-2 \mathrm{~V}$ ) in a $\mathrm{P}$ only extrinsic photoresistor with different traps concentration (Fig. 7a) and trap position (Fig. 7b). Parameters are identical to those reported in Table 2 .

\section{6) Conclusions}

The mechanism of Trap Assisted Photo-Multiplication in vertical organic photoresistor, observed in several previous experimental works, was investigated on a theoretical point of view in this paper, providing several interesting conclusions.

First of all, drift diffusion simulations have confirmed that a gain may occur, essentially in $\mathrm{P}$ only (or $\mathrm{N}$ only) photoresistor, but not in classical photodiodes or intrinsic photoresistor. Our model has allowed to analyze in details the origin of this gain. It turns out that photo-generated carriers, if able to induce a significant modification of electric field, may trigger additional carrier injection from the contacts. This mechanism has been called "gain by injection enhancement". It is responsible for a significant dependency of the photoresistor responsivity with irradiance (where "good" photodiodes have typically a constant responsivity), this effect being more sensitive at low irradiance. If carrier injection is limited, as in photodiodes or in intrinsic photoresistor, the gain remains negligible, but can exceed unity in the case of extrinsic photoresistor. Traps, fixing photogenerated carriers in the active layer, (electrons for $\mathrm{P}$ only devices, or hole for $\mathrm{N}$ only devices) may be an excellent way to induce such additional electric field, even if this mechanism may appear also in absence of traps in principle (however at moderate level). If the traps considered in this work were uniformly distributed in the active layer, it is clear however that further optimization of this mechanism is possible by playing with the localization and the nature of traps.

All these conclusions were confirmed by comparison with experimental $P$ only PCDTBT:PC60BM photoresistors. Interestingly, once material parameters (mobility, traps ...) were extracted by fitting photodiodes experiments, we found that no further adjustment were required to reproduce satisfactorily photoresistor experiments, confirming the predictive nature of our simulations.

In term of device performances however, gain by injection enhancement has several limitations. First, when the gain is achieved by trapping, it is an extremely slow mechanism (depending on the trap energy, limited a hundred of $\mathrm{Hz}$ in the best case). Second, as gain takes interesting value only when dark currents are significant, it cannot achieve very high value of specific detectivity (it cannot reach the photon shot noise limit for instance). In the template material considered in our work, (PCDTBT:PC60BM blends) photoresistor simulations indicate that it was not possible to reach the value of detectivity already obtained in the best experimental results reported using photodiodes. However, it may remain attractive for other technology and material, where the nature of material and contacts does not allow to scale down the dark current of photodiodes. 


\section{7) Supplementary material}

More information about the impact of applied voltage and wavelength is given in the supplementary material.

\section{8) Acknowledgment}

This work has been funded by the French National Research Agency (ANR) through the TAPIR project $\mathrm{N}^{\circ}$ ANR-15-CE24-0024-01.

9) References

${ }^{1}$ G. Dennler, M.C. Scharber, and C.J. Brabec, Adv. Mater. 21, 1323 (2009).

${ }^{2}$ N. Espinosa, R. García-Valverde, and A. Urbina, Sol. Energy Mater. Sol. Cells 95, 1293 (2011).

${ }^{3}$ F.P. García De Arquer, A. Armin, P. Meredith, and E.H. Sargent, Nat. Rev. Mater. 2, 16100 (2017)

${ }^{4}$ S.F. Tedde, P. Büchele, R. Fischer, F. Steinbacher, and O. Schmidt, Tech. Dig. - Int. Electron Devices Meet. IEDM 10.4.1 (2014).

${ }^{5}$ R. Eckstein, N. Strobel, T. Rödlmeier, K. Glaser, U. Lemmer, and G. Hernandez-Sosa, Adv. Opt. Mater. 6, 1701108 (2018).

${ }^{6}$ H. Hoppe, M. Niggemann, C. Winder, J. Kraut, R. Hiesgen, A. Hinsch, D. Meissner, and N.S. Sariciftci, Adv. Funct. Mater. 14, 1005 (2004).

${ }^{7}$ A. Pierre and A.C. Arias, in Tech. Dig. - Int. Electron Devices Meet. IEDM 32.4.1 (2016).

${ }^{8}$ M. Kielar, O. Dhez, G. Pecastaings, A. Curutchet, and L. Hirsch, Sci. Rep. 6, 39201 (2016).

${ }^{9}$ R. Clerc, B. Bouthinon, M. Mohankumar, P. Rannou, J. Vaillant, T. Maindron, B. Racine, Y.F. Chen, L. Hirsch, J.M. Verilhac, A. Pereira, and A. Revaux, in Tech. Dig. - Int. Electron Devices Meet. IEDM 14.6.1 (2017)

10 M. Vollmer and K.P. Möllmann, Infrared Thermal Imaging: Fundamentals, Research and Applications, (John Wiley \& Sons, New York, 2017).

${ }^{11}$ R. Nie, X. Deng, L. Feng, G. Hu, Y. Wang, G. Yu, and J. Xu, Small 13, 1603260 (2017).

12 D. Yang, X. Zhou, Y. Wang, A. Vadim, S.M. Alshehri, T. Ahamad, and D. Ma, J. Mater. Chem. C 4, 2160 (2016).

${ }^{13}$ R. Dong, Y. Fang, J. Chae, J. Dai, Z. Xiao, Q. Dong, Y. Yuan, A. Centrone, X.C. Zeng, and J. Huang, Adv. Mater. 27, 1912 (2015).

${ }^{14}$ Y. Fu, Q. Song, T. Lin, Y. Wang, X. Sun, Z. Su, B. Chu, F. Jin, H. Zhao, W. Li, and C.S. Lee, Org. Electron. 51, 200 (2017).

${ }^{15}$ T. Miyasaka, Chem. Lett. 44, 720 (2015).

${ }^{16}$ L. Li, F. Zhang, J. Wang, Q. An, Q. Sun, W. Wang, J. Zhang, and F. Teng, Sci. Rep. 5, 9181 (2015).

${ }^{17}$ X. Li, S. Wang, Y. Xiao, and X. Li, J. Mater. Chem. C 4, 5584 (2016).

${ }^{18}$ L. Li, F. Zhang, W. Wang, Q. An, J. Wang, Q. Sun, and M. Zhang, ACS Appl. Mater. Interfaces, 7, 5890 (2015)

${ }^{19}$ I.H. Campbell and B.K. Crone, J. Appl. Phys. 101, 024502 (2007). 
${ }^{20}$ F. Guo, B. Yang, Y. Yuan, Z. Xiao, Q. Dong, Y. Bi, and J. Huang, Nat. Nanotechnol. 7, 798 (2012).

${ }^{21}$ J.W. Lee, D.Y. Kim, and F. So, Adv. Funct. Mater. 25, 1233 (2015).

${ }^{22}$ G. Konstantatos and E.H. Sargent, Appl. Phys. Lett. 91, 173505 (2007).

${ }^{23}$ A. De lacovo, C. Venettacci, L. Colace, L. Scopa, and S. Foglia, Sci. Rep. 6, 37913 (2016).

${ }^{24}$ T. Kyu An, C. Eon Park, and D. Sung Chung, Appl. Phys. Lett. 102, 193306 (2013).

${ }^{25}$ H. Wei, Y. Fang, Y. Yuan, L. Shen, and J. Huang, Adv. Mater. 27, 4975 (2015).

${ }^{26}$ D.S. Chung, Y. Rho, M. Ree, S.K. Kwon, and Y.H. Kim, ACS Appl. Mater. Interfaces 4, 4758 (2012).

${ }^{27}$ L. Li, F. Zhang, W. Wang, Y. Fang, and J. Huang, Phys. Chem. Chem. Phys. 17, 30712 (2015).

${ }^{28}$ W.T. Hammond, J.P. Mudrick, and J. Xue, J. Appl. Phys. 116, 214501 (2014).

${ }^{29}$ S. M. Sze and K. K. Ng, Physics of Semiconductor Devices, (John Wiley \& Sons, New York, 2006).

${ }^{30}$ R.H. Bube, Photoconductivity of Solids (John Wiley \& Sons, New York, 1960).

${ }^{31}$ L. Koster, E. Smits, V. Mihailetchi, and P. Blom, Phys. Rev. B 72, 085205 (2005).

32 Y. Zhou, C. Fuentes-Hernandez, J. Shim, J. Meyer, A.J. Giordano, H. Li, P. Winget, T. Papadopoulos, H. Cheun, J. Kim, M. Fenoll, A. Dindar, W. Haske, E. Najafabadi, T.M. Khan, H. Sojoudi, S. Barlow, S. Graham, J.-L. Brédas, S.R. Marder, A. Kahn, and B. Kippelen, Science 336, 327 (2012).

${ }^{33}$ R. Häusermann, E. Knapp, M. Moos, N. a. Reinke, T. Flatz, and B. Ruhstaller, J. Appl. Phys. 106, 104507 (2009).

${ }^{34}$ D.J.D. Moet, P. de Bruyn, J.D. Kotlarski, and P.W.M. Blom, Org. Electron. 11, 1821 (2010).

35 S. Altazin, R. Clerc, R. Gwoziecki, G. Pananakakis, G. Ghibaudo, and C. Serbutoviez, Appl. Phys. Lett. 99, 143301 (2011).

36 A. G. Fluxim, SETFOS: Semiconducting emissive thin film optics simulator software, http://www.fluxim.com.

${ }^{37}$ M. Kielar, M. Daanoune, O. François-Martin, B. Flament, O. Dhez, A.K. Pandey, S. Chambon, R. Clerc, and L. Hirsch, Adv. Electron. Mater. 4, 1700526 (2018).

${ }^{38}$ R.A. Street, Phys. Rev. B 84, 075208 (2011).

${ }^{39}$ S. Alem, T.Y. Chu, S.C. Tse, S. Wakim, J. Lu, R. Movileanu, Y. Tao, F. Bélanger, D. Désilets, S. Beaupré, M. Leclerc, S. Rodman, D. Waller, and R. Gaudiana, Org. Electron. 12, 1788 (2011).

$40 \mathrm{~J}$. Schafferhans, A. Baumann, A. Wagenpfahl, C. Deibel, and V. Dyakonov, Org. Electron. 11, 1693 (2010).

${ }^{41}$ A. Seemann, T. Sauermann, C. Lungenschmied, O. Armbruster, S. Bauer, H.-J. Egelhaaf, and J. Hauch, Sol. Energy 85, 1238 (2011).

42 M.M. Mandoc, W. Veurman, L.J. a. Koster, B. de Boer, and P.W.M. Blom, Adv. Funct. Mater. 17, 2167 (2007).

${ }^{43}$ S.R. Cowan, W.L. Leong, N. Banerji, G. Dennler, and A.J. Heeger, Adv. Funct. Mater. 21, 3083 (2011).

${ }^{44}$ A.B. Djurišić, T. Fritz, and K. Leo, Opt. Commun. 183, 123 (2000). 


\section{Appendix A : Derivation of the formula of gain by injection enhancement}

According to the drift diffusion model, the electron and hole carrier flux $\left(\mathrm{m}^{-2} \mathrm{~s}^{-1}\right)$ at each point $\mathrm{x}$ are given by:

$$
\begin{aligned}
& \phi_{n}=\frac{k T}{e} \mu_{n}\left(-\frac{d n}{d x}+\frac{e n}{k T} \frac{d V}{d x}\right) \\
& \phi_{p}=\frac{k T}{e} \mu_{p}\left(-\frac{d p}{d x}-\frac{e p}{k T} \frac{d V}{d x}\right)
\end{aligned}
$$

where $\mu_{n}$ and $\mu_{p}$ are the electrons and holes mobility, $k$ the Boltzmann constant, $T$ the temperature, $\mathrm{n}$ and $\mathrm{p}$ the carrier concentration (electrons and holes respectively), e the elementary electron charge and $\mathrm{V}$ the potential.

The conservation equation of electrons and holes leads to :

$$
\frac{d \phi_{n}}{d x}=\frac{d \phi_{p}}{d x}=G-R
$$

where $G(x)$ and $R(x)$ are respectively the electron-hole carrier generation and recombination rates. These equations ensure the conservation of the total current density jillum $\left(\mathrm{Am}^{-2}\right)$, as :

$$
\frac{d j}{d x}=\frac{d}{d x}\left(-e \phi_{n}+e \phi_{p}\right)=0
$$

The conservation equation of electron can be written ${ }^{29}$ in the following form by multiplication of each side par $\exp (-\mathrm{eV} / \mathrm{kT})$ :

$$
\phi_{n} \exp \left(-\frac{e V}{k T}\right)=-\frac{k T}{e} \mu_{n} \frac{d}{d x}\left(n \exp \left(-\frac{e V}{k T}\right)\right)
$$

By integrating this equation through the entire structure and using the classical Schottky boundary conditions (the same boundary condition implemented in the simulation), the right-hand side simplifies and the conservation equation becomes:

$$
\int_{0}^{L} \phi_{n}(x) \exp \left(-\frac{e V(x)}{k T}\right) d x=-\frac{k T}{e} \mu_{n}\left[n_{L} \exp \left(-\frac{e V_{L}}{k T}\right)-n_{0} \exp \left(-\frac{e V_{0}}{k T}\right)\right]
$$

where $L$ is the active layer thicknesses, $n_{L}=n(x=L)$ (resp. $n_{0}=n(x=0)$ ) is the electron concentration at the left (resp. right) contact, and $V_{L}-V_{0}=V_{a}$, the applied voltage.

Therefore, thanks to the boundary conditions, this integral has the same value in dark and illumination conditions. Consequently,

$$
\int_{0}^{L} \phi_{n}(x) \exp \left(-\frac{e V(x)}{k T}\right) d x=\int_{0}^{L} \phi_{n d}(x) \exp \left(-\frac{e V_{d}(x)}{k T}\right) d x
$$

where $V_{d}(x)$ is the potential profile in dark condition, $\phi_{n d}(x)$ the flux of electrons in dark.

According Eq.(A3), $\phi_{n}(x)$ can be replaced by:

Where :

$$
\phi_{n}(x)=\phi_{G}(x)-\phi_{R}(x)+\tilde{\phi}_{n}
$$

$$
\begin{aligned}
& \phi_{G}(x)=\int_{0}^{x} G\left(x^{\prime}\right) d x^{\prime} \\
& \phi_{R}(x)=\int_{0}^{x} R\left(x^{\prime}\right) d x^{\prime}
\end{aligned}
$$

$\tilde{\phi}_{n}$ is a constant, that can be related to the current density. 
Note that $\phi_{G}(L)$ is the total number of electrons holes pairs generated, thus the number of photons absorbed.

In dark condition, we have :

Replacing Eq. (A7) into Eq. (A8) leads to :

$$
\phi_{n d}(x)=\tilde{\phi}_{n d}-\phi_{R d}(x)
$$

$$
\begin{aligned}
\tilde{\phi}_{n} \int_{0}^{L} \exp \left(-\frac{e V(x)}{k T}\right) d x \\
\quad=\tilde{\phi}_{n d} \int_{0}^{L} \exp \left(-\frac{e V_{d}(x)}{k T}\right) d x-\int_{0}^{L}\left[\phi_{G}(x)-\phi_{R}(x)\right] \exp \left(-\frac{e V(x)}{k T}\right) d x \\
-\int_{0}^{L} \phi_{R d}(x) \exp \left(-\frac{e V_{d}(x)}{k T}\right) d x
\end{aligned}
$$

Which can be rewritten as :

$$
\begin{gathered}
\tilde{\phi}_{n}-\tilde{\phi}_{n d}=\tilde{\phi}_{n d}\left(\frac{\int_{0}^{L} \exp \left(-\frac{e V_{d}(x)}{k T}\right) d x}{\int_{0}^{L} \exp \left(-\frac{e V(x)}{k T}\right) d x}-1\right)-\frac{\int_{0}^{L}\left[\phi_{G}(x)-\phi_{R}(x)\right] \exp \left(-\frac{e V(x)}{k T}\right) d x}{\int_{0}^{L} \exp \left(-\frac{e V(x)}{k T}\right) d x} \\
-\frac{\int_{0}^{L} \phi_{R d}(x) \exp \left(-\frac{e V_{d}(x)}{k T}\right) d x}{\int_{0}^{L} \exp \left(-\frac{e V(x)}{k T}\right) d x}
\end{gathered}
$$

This equation can be compacted introducing the following notation:

where :

$$
\tilde{\phi}_{n}-\tilde{\phi}_{n d}=\beta_{n} \tilde{\phi}_{n d}-<\phi_{G}(x)-\phi_{R}(x)>_{n i}-<\phi_{R d}(x)>_{n d}
$$

$$
\begin{gathered}
\beta_{n}=\frac{\int_{0}^{L} \exp \left(-\frac{e V_{d}(x)}{k T}\right) d x}{\int_{0}^{L} \exp \left(-\frac{e V(x)}{k T}\right) d x}-1 \\
<\phi_{G}(x)-\phi_{R}(x)>_{n i}=\frac{\int_{0}^{L}\left[\phi_{G}(x)-\phi_{R}(x)\right] \exp \left(-\frac{e V(x)}{k T}\right) d x}{\int_{0}^{L} \exp \left(-\frac{e V(x)}{k T}\right) d x} \\
<\phi_{R d}(x)>_{n d}=\frac{\int_{0}^{L} \phi_{R d}(x) \exp \left(-\frac{e V_{d}(x)}{k T}\right) d x}{\int_{0}^{L} \exp \left(-\frac{e V(x)}{k T}\right) d x}
\end{gathered}
$$

Using the same procedure for holes conservation equation, we found :

$$
\begin{gathered}
\tilde{\phi}_{p}-\tilde{\phi}_{p d}=\beta_{p} \tilde{\phi}_{p d}-<\phi_{G}(x)-\phi_{R}(x)>_{p i}-<\phi_{R d}(x)>_{p d} \\
\beta_{p}=\frac{\int_{0}^{L} \exp \left(\frac{e V_{d}(x)}{k T}\right) d x}{\int_{0}^{L} \exp \left(\frac{e V(x)}{k T}\right) d x}-1 \\
<\phi_{G}(x)-\phi_{R}(x)>_{p i}=\frac{\int_{0}^{L}\left[\phi_{G}(x)-\phi_{R}(x)\right] \exp \left(\frac{e V(x)}{k T}\right) d x}{\int_{0}^{L} \exp \left(\frac{e V(x)}{k T}\right) d x}
\end{gathered}
$$




$$
<\phi_{R d}(x)>_{p d}=\frac{\int_{0}^{L} \phi_{R d}(x) \exp \left(\frac{e V_{d}(x)}{k T}\right) d x}{\int_{0}^{L} \exp \left(\frac{e V(x)}{k T}\right) d x}
$$

According Eq. (A4), the constant current density under illumination is given by :

$$
j_{\text {illum }}=-e \phi_{n}(x)+e \phi_{p}(x)=-e \phi_{n}(0)+e \phi_{p}(0)=-e \tilde{\phi}_{n}+e \tilde{\phi}_{p}
$$

Similarly, in dark condition:

$$
j_{d a r k}=-e \phi_{n d}(x)+e \phi_{p d}(x)=-e \phi_{n d}(0)+e \phi_{p d}(0)=-e \tilde{\phi}_{n d}+e \tilde{\phi}_{p d}
$$

Thus, the photocurrent $j_{\text {illum }}-j_{\text {dark }}$ is given by :

$$
\begin{aligned}
\left(j_{\text {illum }}-j_{\text {dark }}\right) / e & \\
& =-\left[\beta_{n} \tilde{\phi}_{n d}-<\phi_{G}(x)-\phi_{R}(x)>_{n i}-<\phi_{R d}(x)>_{n d}\right] \\
& +\left[\beta_{p} \tilde{\phi}_{p d}-<\phi_{G}(x)-\phi_{R}(x)>_{p i}-<\phi_{R d}(x)>_{p d}\right]
\end{aligned}
$$

The previous equation can compacted as :

$$
\frac{j_{\text {illum }}-j_{\text {dark }}}{e}=-\beta_{n} \tilde{\phi}_{n d}+\beta_{p} \tilde{\phi}_{p d}+<\phi_{G}(x)-\phi_{R}(x)>_{i}-<\phi_{R d}(x)>_{d}
$$

With :

$$
\begin{gathered}
<\phi_{G}(x)-\phi_{R}(x)>_{i} \\
=-\frac{\int_{0}^{L}\left[\phi_{G}(x)-\phi_{R}(x)\right] \exp \left(\frac{e V(x)}{k T}\right) d x}{\int_{0}^{L} \exp \left(\frac{e V(x)}{k T}\right) d x} \\
+\frac{\int_{0}^{L}\left[\phi_{G}(x)-\phi_{R}(x)\right] \exp \left(-\frac{e V(x)}{k T}\right) d x}{\int_{0}^{L} \exp \left(-\frac{e V(x)}{k T}\right) d x} \\
<\phi_{R d}(x)>_{d}=\frac{\int_{0}^{L} \phi_{R d}(x) \exp \left(\frac{e V_{d}(x)}{k T}\right) d x}{\int_{0}^{L} \exp \left(\frac{e V(x)}{k T}\right) d x}-\frac{\int_{0}^{L} \phi_{R d}(x) \exp \left(-\frac{e V_{d}(x)}{k T}\right) d x}{\int_{0}^{L} \exp \left(-\frac{e V(x)}{k T}\right) d x}
\end{gathered}
$$

Finally, the Gain is defined as the number of photo electrons collected, divided by the number of photon absorbed:

$$
\text { Gain }=\frac{\left|j_{\text {illum }}-j_{\text {dark }}\right| / e}{\phi_{G}(\mathrm{~L})}
$$

Using Eq. (A25), we obtain the following equation for the Gain :

$$
\text { Gain }=\left|\frac{-\beta_{n} \tilde{\phi}_{n d}+\beta_{p} \tilde{\phi}_{p d}}{\phi_{G}(\mathrm{~L})}+\frac{<\phi_{G}(x)-\phi_{R}(x)>_{i}-<\phi_{R d}(x)>_{d}}{\phi_{G}(\mathrm{~L})}\right|
$$


a)

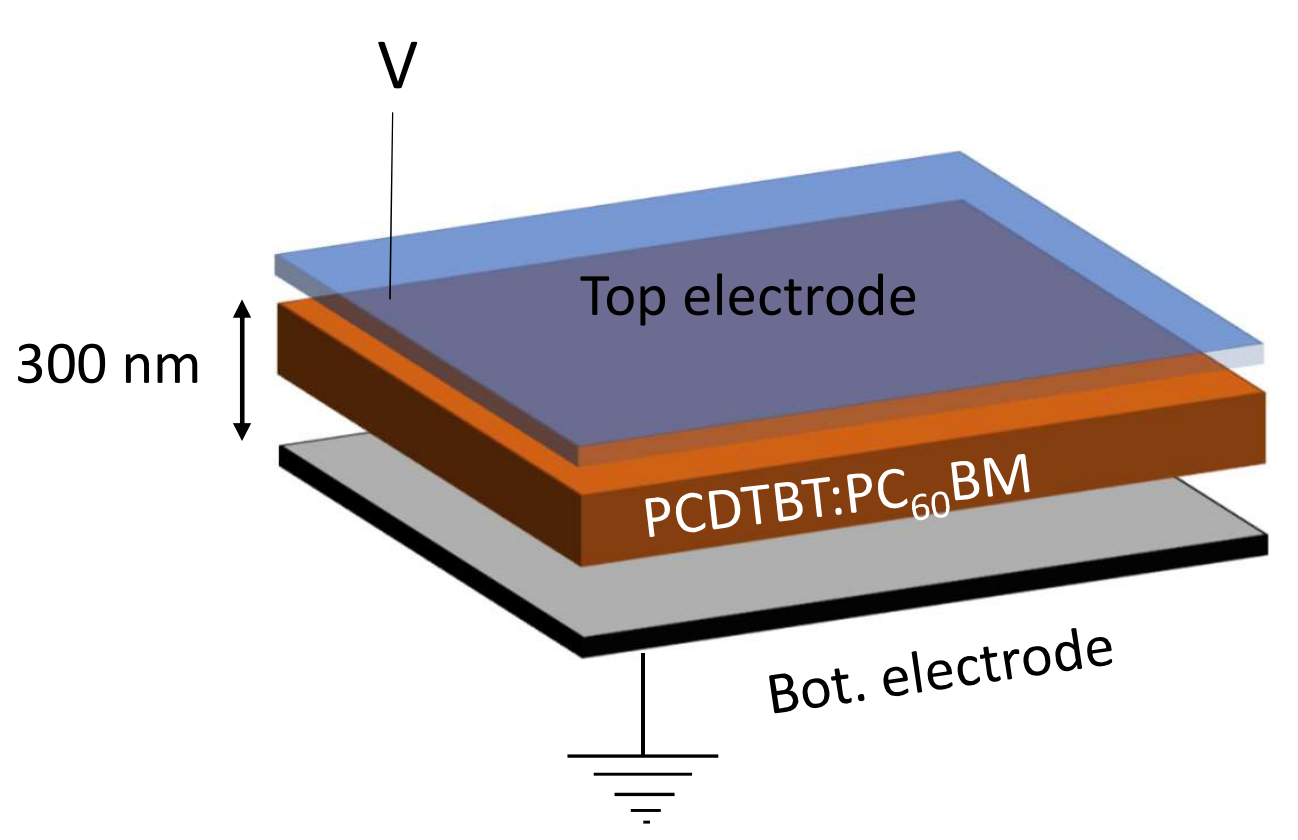


b)
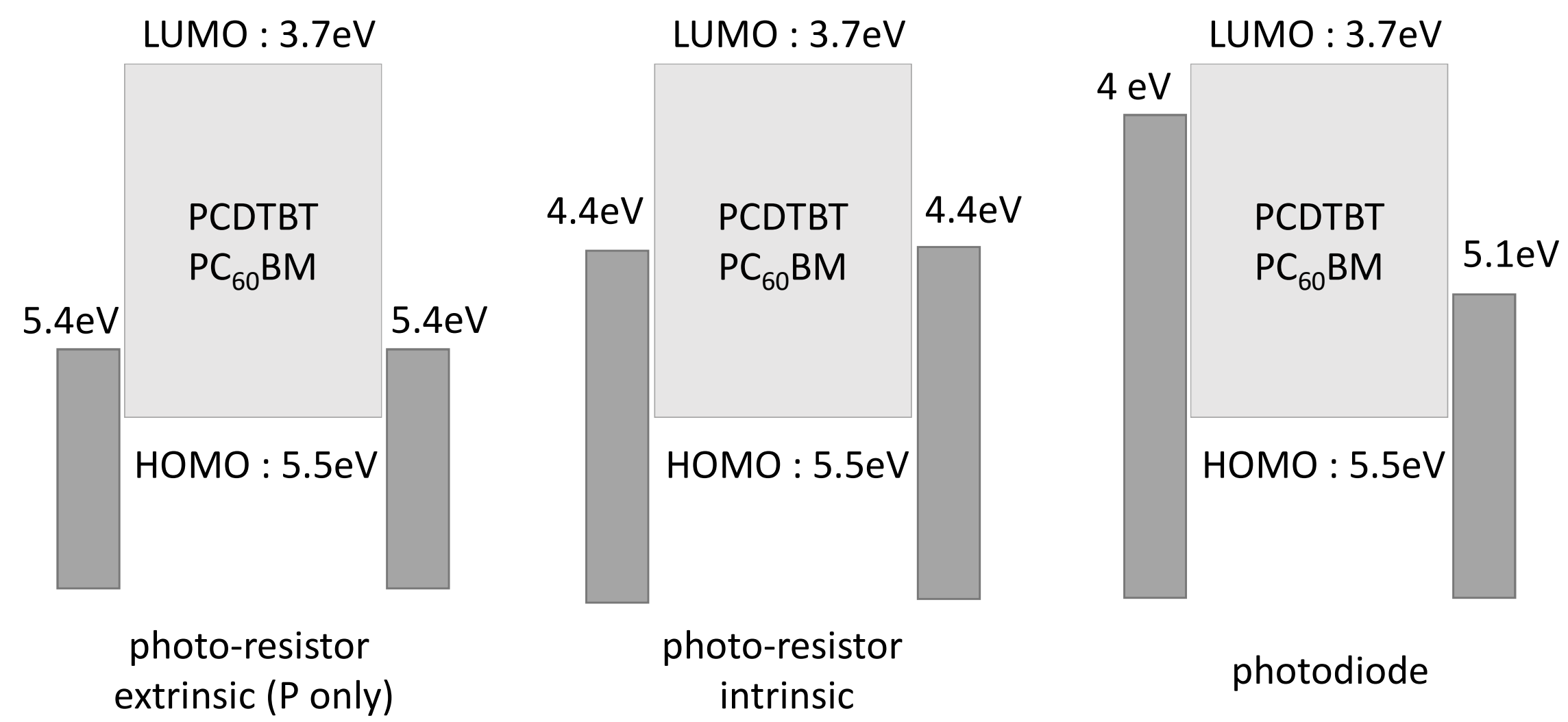


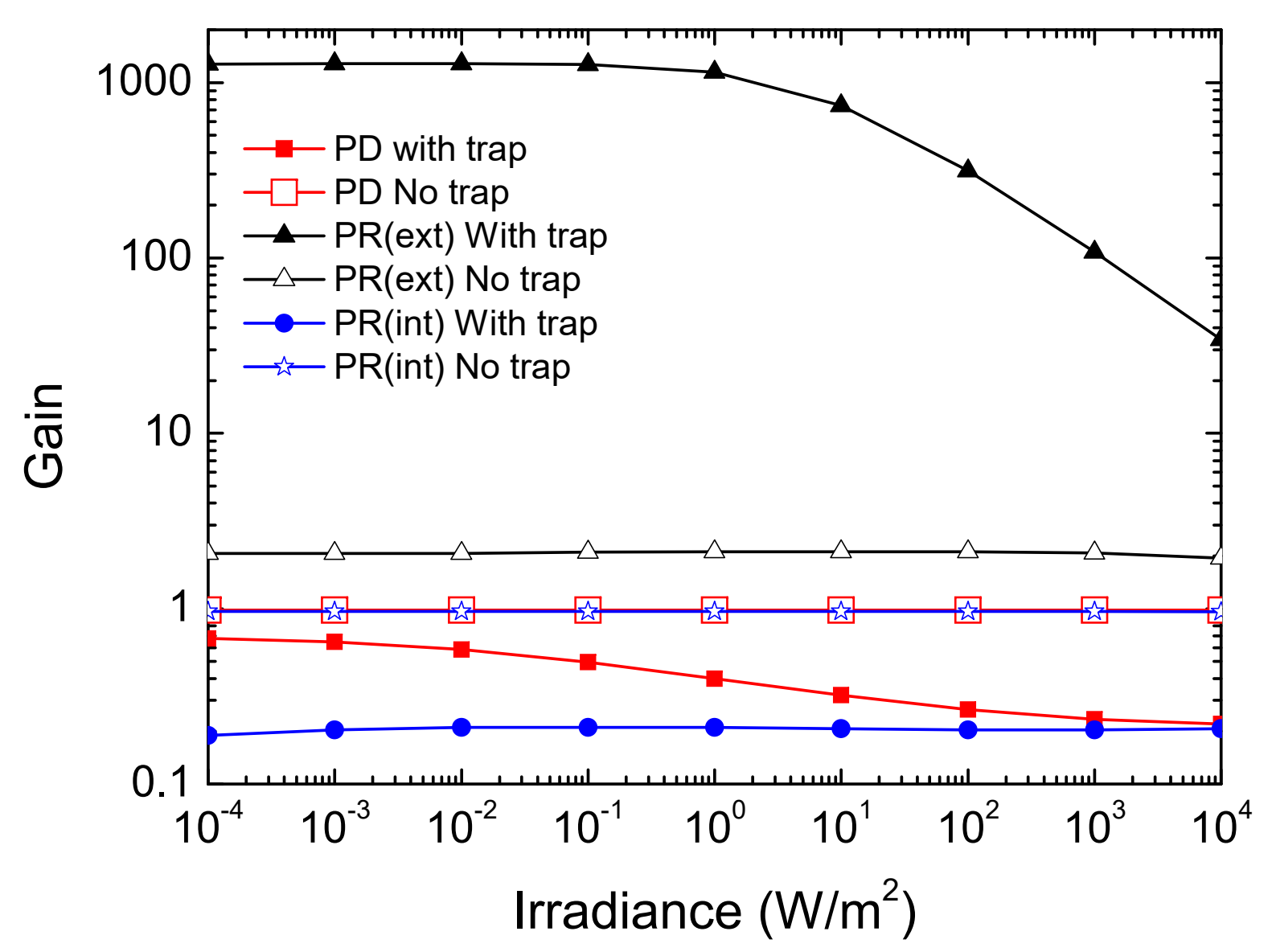




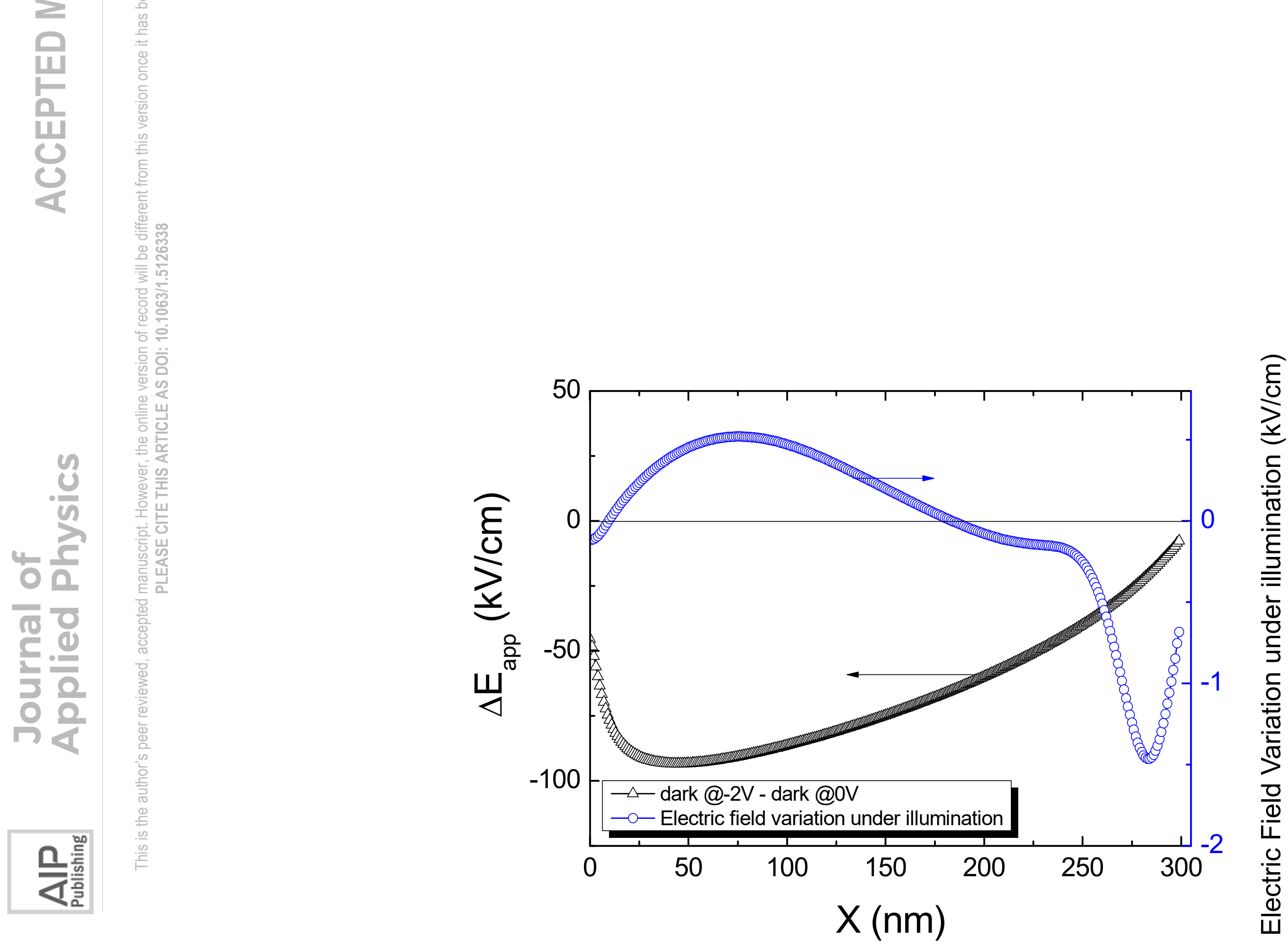


(a)

simulation with trap

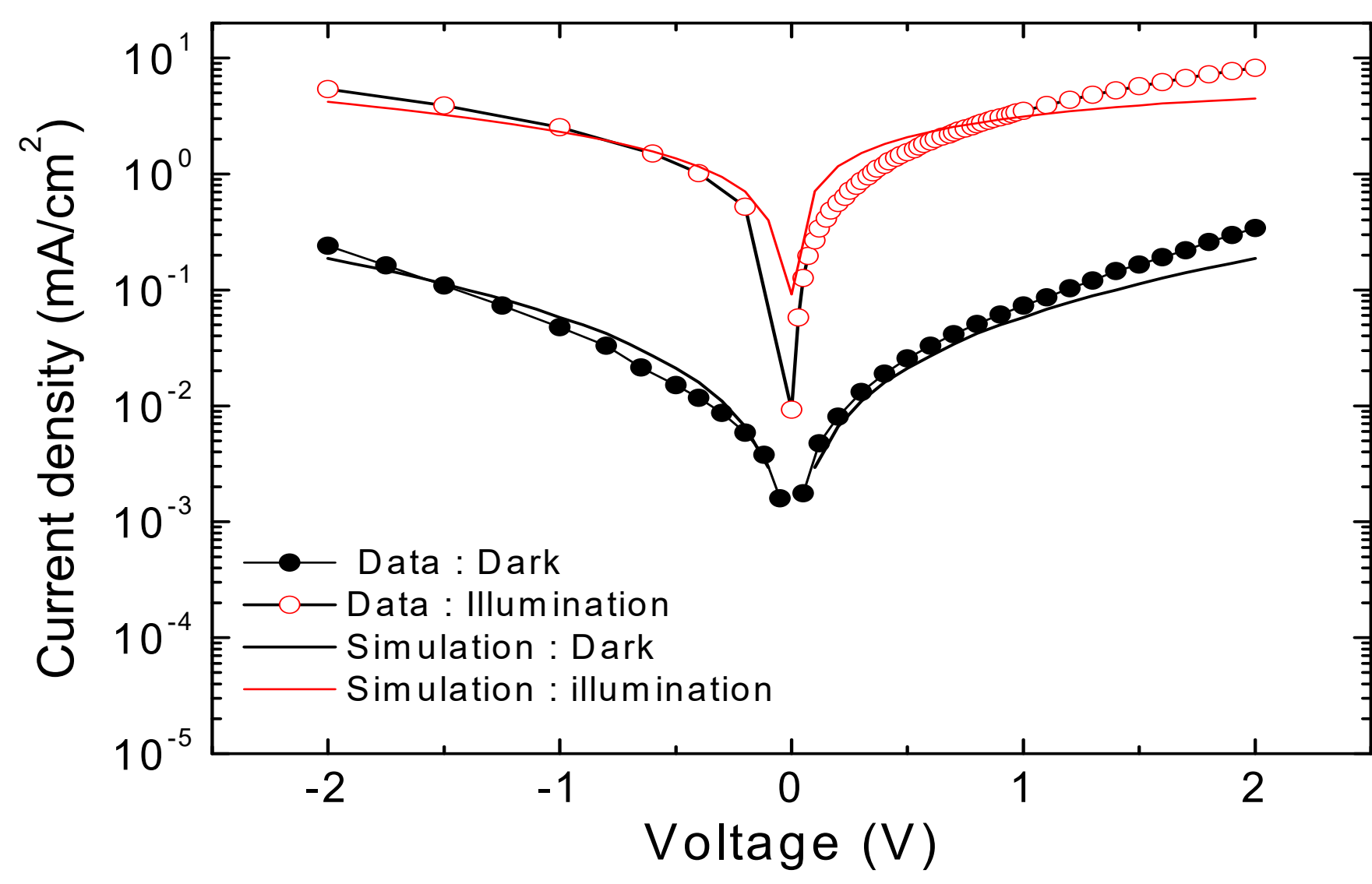




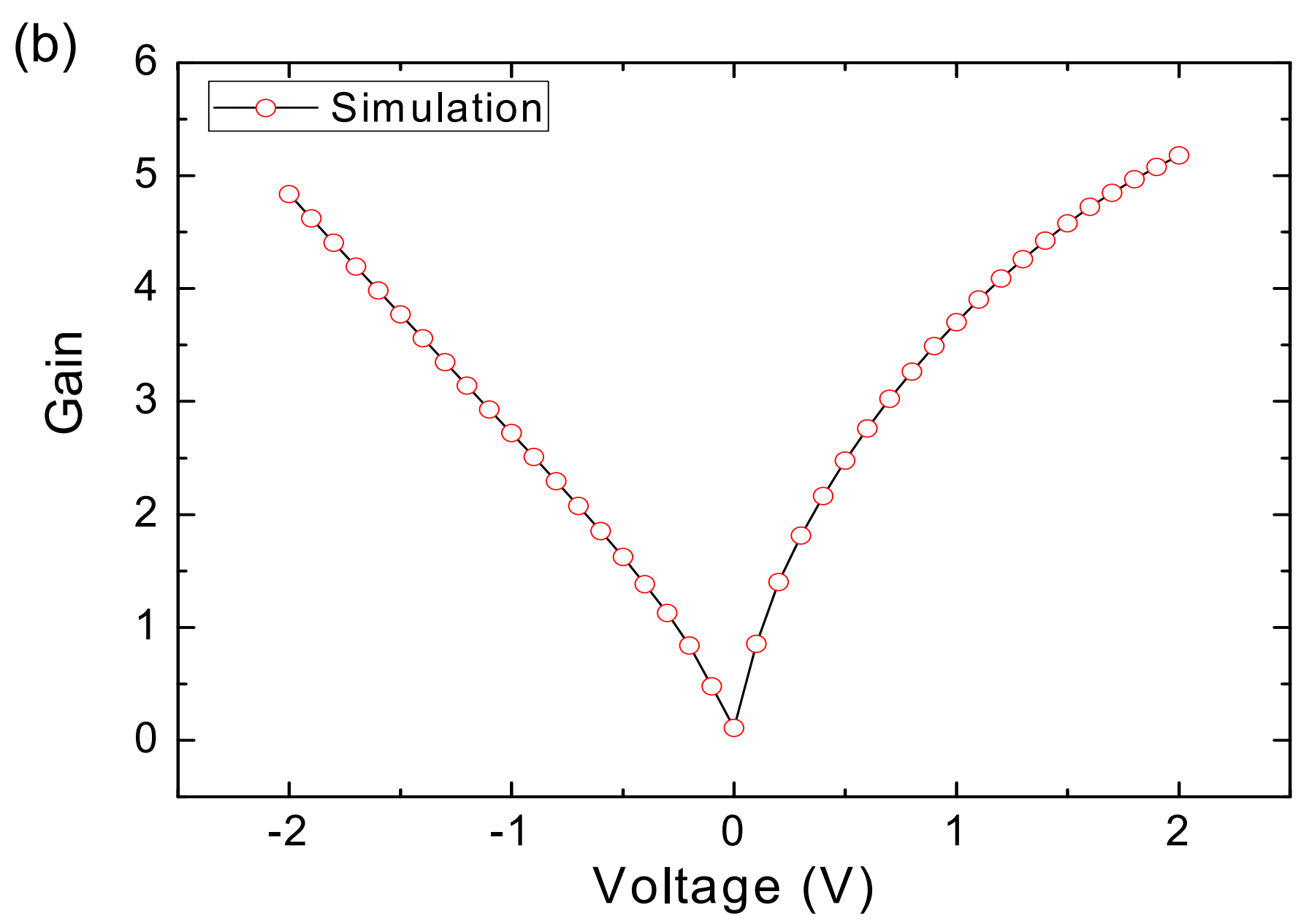


(a)

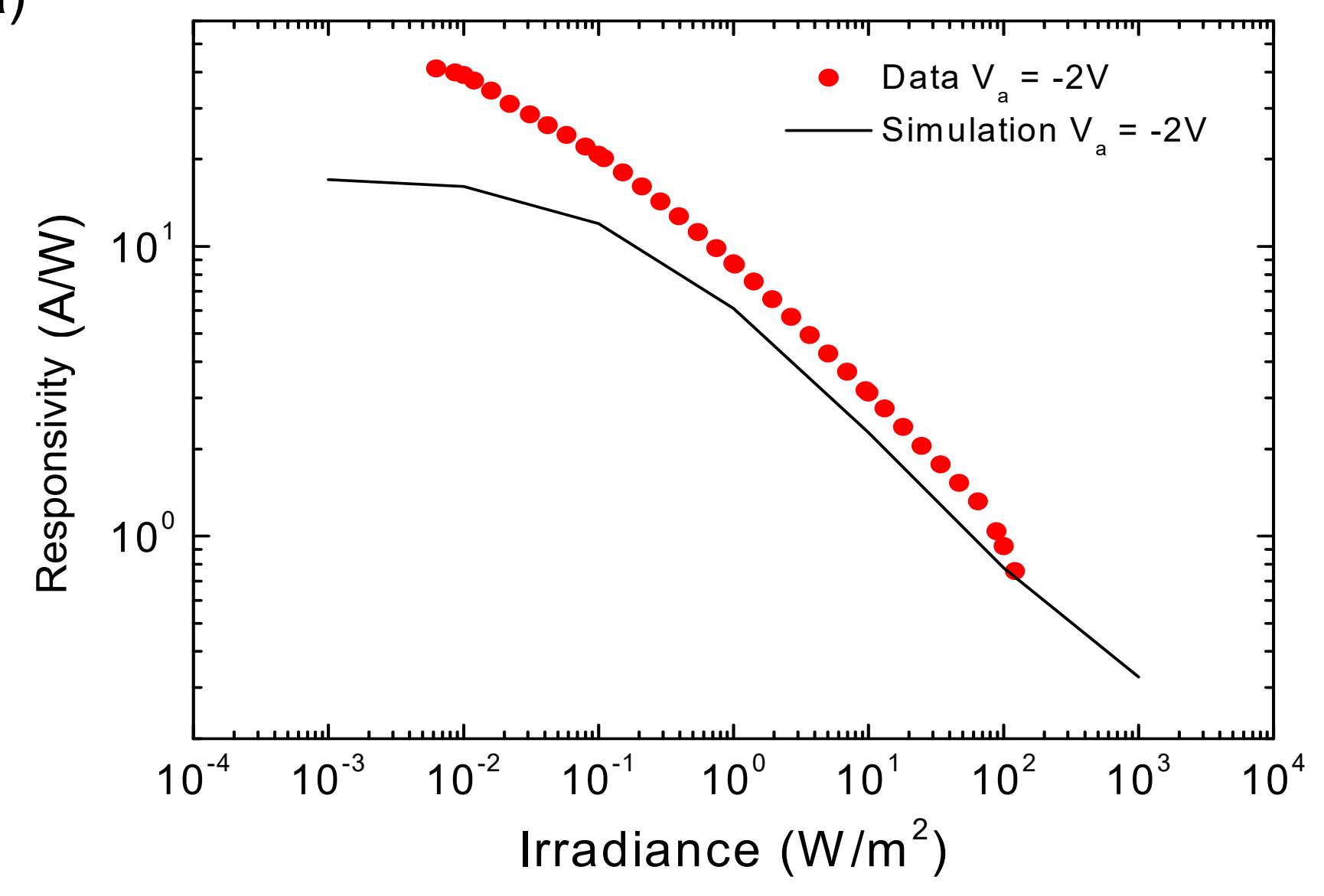


(b)

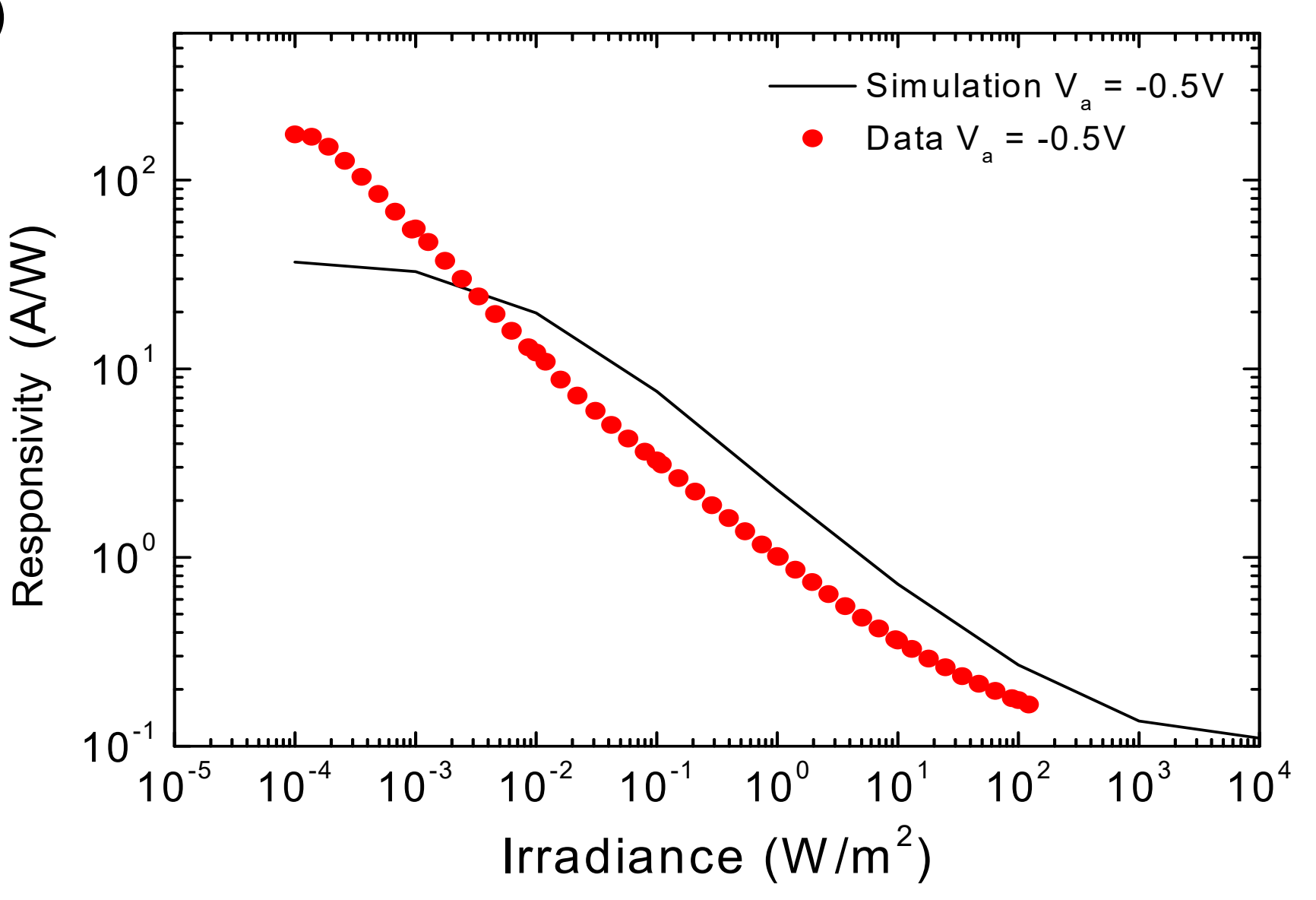




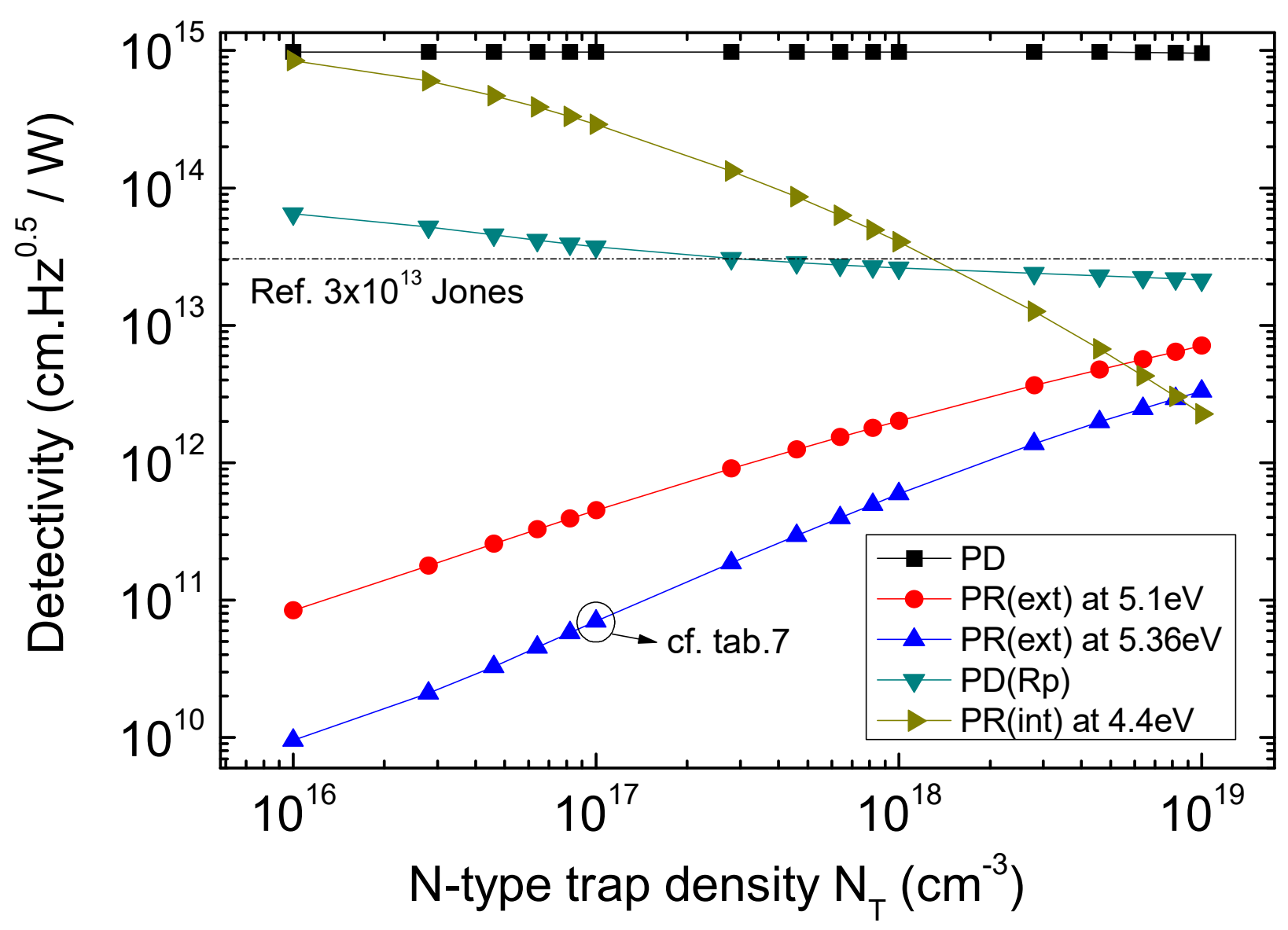


(a)

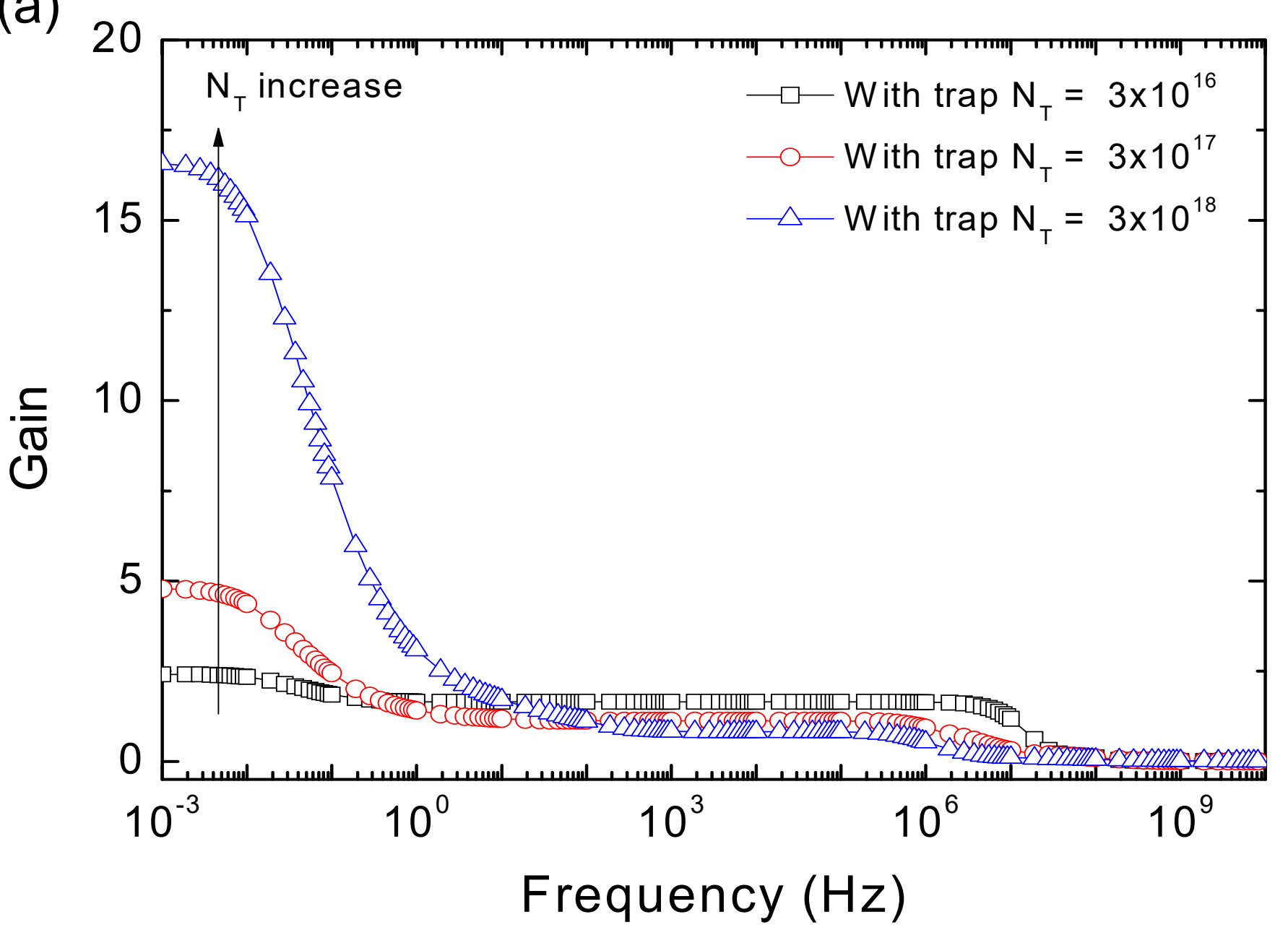


(b)

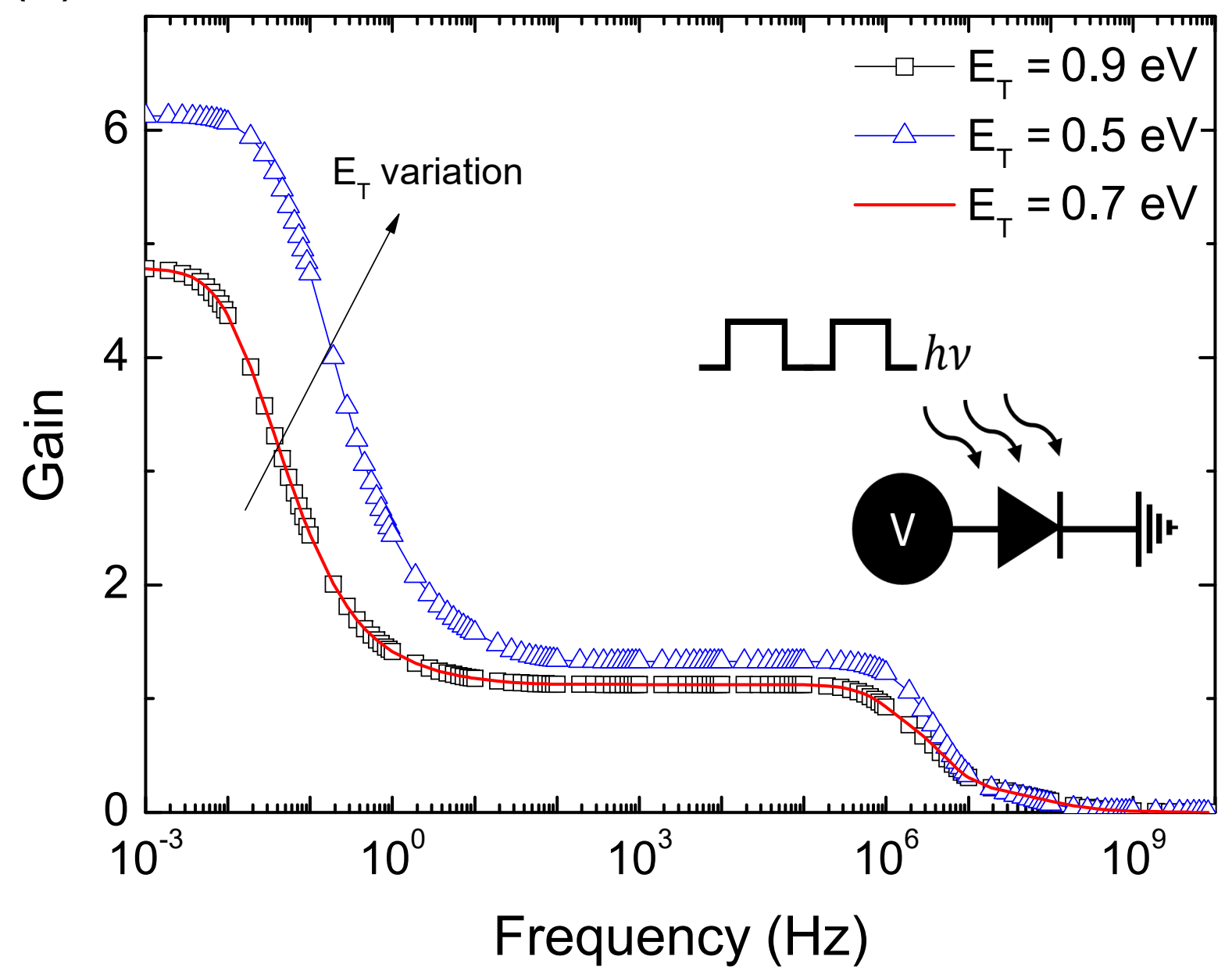

